\title{
The Extremely Luminous Quasar Survey in the Sloan Digital Sky Survey Footprint. III. The South Galactic Cap Sample and the Quasar Luminosity Function at Cosmic Noon
}

\author{
Jan-Torge Schindler ${ }^{1,2}$ (D), Xiaohui Fan ${ }^{1}$ (D), Ian D. McGreer ${ }^{1}$ (i), Jinyi Yang ${ }^{1}$ (iD, Feige Wang ${ }^{3}$ (D), Richard Green ${ }^{1}$,

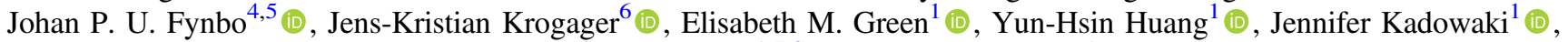 \\ Anna Patej ${ }^{1}$, Ya-Lin $\mathrm{Wu}^{1,7,8}$ (10), and Minghao Yue ${ }^{1}$ (1) \\ ${ }^{1}$ Steward Observatory, University of Arizona, 933 North Cherry Avenue, Tucson, AZ 85721, USA \\ ${ }^{2}$ Max-Planck Institute for Astronomy, Königstuhl 17, D-69117 Heidelberg, Germany \\ ${ }^{3}$ Department of Physics, University of California Santa Barbara, Santa Barbara, CA 93106-9530, USA \\ ${ }^{4}$ Cosmic Dawn Center (DAWN), Niels Bohr Institute, University of Copenhagen, Juliane Maries Vej 30, DK-2100 Copenhagen Ø, Denmark \\ ${ }_{5}^{5}$ DTU-Space, Technical University of Denmark, Elektrovej 327, DK-2800 Kgs. Lyngby, Denmark \\ ${ }^{6}$ Institut d'Astrophysique de Paris, CNRS-UPMC, UMR7095, 98bis bd Arago, F-75014 Paris, France \\ ${ }^{7}$ Department of Astronomy, University of Texas at Austin, 2515 Speedway, Stop C1400, Austin, TX 78712-1205, USA \\ Received 2018 September 23; revised 2018 December 10; accepted 2018 December 10; published 2019 February 5
}

\begin{abstract}
We have designed the Extremely Luminous Quasar Survey (ELQS) to provide a highly complete census of unobscured UV-bright quasars during the cosmic noon, $z=2.8-5.0$. Here we report the discovery of 70 new quasars in the ELQS South Galactic Cap (ELQS-S) quasar sample, doubling the number of known extremely luminous quasars in $4237.3 \mathrm{deg}^{2}$ of the Sloan Digital Sky Survey footprint. These observations conclude the ELQS and we present the properties of the full ELQS quasar catalog, containing 407 quasars over $11,838.5 \mathrm{deg}^{2}$. Our novel ELQS quasar selection strategy resulted in unprecedented completeness at the bright end and allowed us to discover 109 new quasars in total. This marks an increase of $\sim 36 \%$ (109/298) in the known population at these redshifts and magnitudes, while we further are able to retain a selection efficiency of $\sim 80 \%$. On the basis of 166 quasars from the full ELQS quasar catalog, which adhere to the uniform criteria of the Two Micron All Sky Survey point source catalog, we measure the bright-end quasar luminosity function (QLF) and extend it one magnitude brighter than previous studies. Assuming a single power law with exponential density evolution for the functional form of the QLF, we retrieve the best-fit parameters from a maximum likelihood analysis. We find a steep brightend slope of $\beta \approx-4.1$, and we can constrain the bright-end slope to $\beta \leqslant-3.4$ with $99 \%$ confidence. The density is well modeled by the exponential redshift evolution, resulting in a moderate decrease with redshift $(\gamma \approx-0.4)$.
\end{abstract}

Key words: galaxies: active - galaxies: high-redshift - galaxies: nuclei - quasars: general

Supporting material: machine-readable table

\section{Introduction}

Quasars are the most luminous non-transient light sources in the universe. A quasar's emission emanates from the accretion disk around a rapidly growing supermassive black hole $(\mathrm{SMBH})$ at the center of a galaxy. The study of quasars provides crucial insight into the formation and evolution of galaxies because the mass of the SMBH and properties of the host galaxy show strong correlations (see Kormendy \& Ho 2013, for a review). Quasars discovered within the first billion years of the universe (Mortlock et al. 2011; Bañados et al. 2018) probe the era of reionization and place strong constraints on the formation and growth of SMBHs. As bright background sources, they have also furthered our understanding of the nature and evolution of the intervening intergalactic medium (Simcoe et al. 2004; Prochaska et al. 2005; Worseck \& Prochaska 2011).

Our understanding of the cosmic growth of SMBHs relies strongly on the demographics of the quasar population, with the quasar luminosity function (QLF) being one of the most fundamental probes. The QLF is best described by a broken double power law (DPL) (Boyle et al. 1988, 2000; Pei 1995), characterized by a faint-end slope, a bright-end slope, an overall normalization, and a break luminosity, where the slopes change.

\footnotetext{
${ }^{8} 51$ Pegasi b Fellow.
}

The faint-end slope is generally flatter than the bright-end slope, and all four parameters possibly change with redshift.

Large-volume spectroscopic surveys, such as the Sloan Digital Sky Survey (SDSS; York et al. 2000), the Baryon Oscillation Spectroscopic Survey (BOSS; Eisenstein et al. 2011; Dawson et al. 2013), and the extended BOSS (eBOSS; Dawson et al. 2016; Blanton et al. 2017), built the largest optical quasar sample to date. This allowed the QLF of UV-bright unobscured quasars to be tightly constrained over a wide range of luminosities and redshifts $(0.3 \lesssim z \lesssim 5)$. At higher redshifts $(z \geqslant 5)$, specifically targeted surveys have constrained the QLF (e.g., Jiang et al. 2008; McGreer et al. 2013; Yang et al. 2016).

At intermediate redshifts $(z=2.8-4.5)$ there has been a standing debate in the literature on the evolution of the brightend slope. Some earlier studies suggested that the bright-end slope would flatten with redshift (Koo \& Kron 1988; Schmidt et al. 1995; Fan et al. 2001; Richards et al. 2006). However, more recent estimates of the QLF seem to indicate that the bright-end slope remains steep up to the highest redshifts (Jiang et al. 2008; Croom et al. 2009; Willott et al. 2010; McGreer et al. 2013; Yang et al. 2016)

This is the third paper in a series presenting the Extremely Luminous Quasar Survey (ELQS), a spectroscopic survey focused on the bright end $\left(m_{i} \leqslant 18.0, M_{1450}<-27\right)$ of the UV-bright type-I quasar distribution at $z \geqslant 2.8$. 


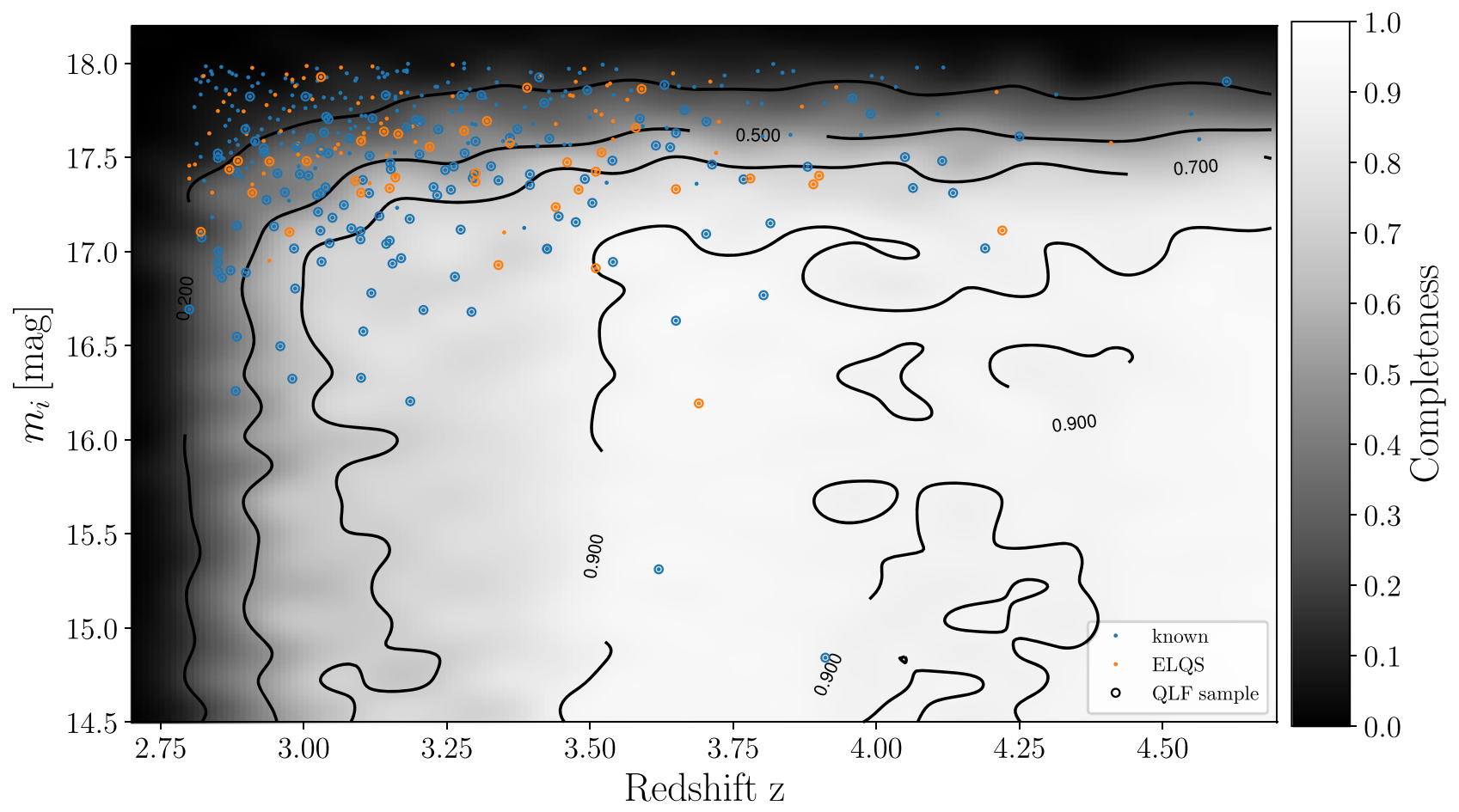

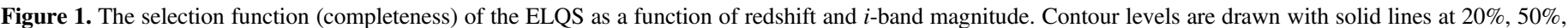

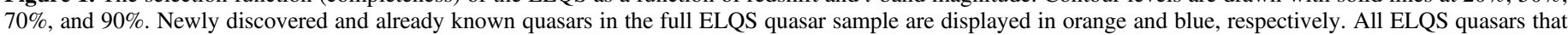
are part of the QLF sample are highlighted.

The first paper (Schindler et al. 2017, hereafter Paper I) discussed the incompleteness of the SDSS spectroscopic quasar survey and BOSS for very bright quasars at these redshifts and showcased our novel quasar selection method.

In the second paper of this series (Schindler et al. 2018, hereafter Paper II) we presented the ELQS quasar sample in the North Galactic Cap (ELQS-N; 90 $<$ R.A. $<270^{\circ}$ ) and a first estimate of the bright-end QLF.

This work presents the final ELQS quasar catalog, covering the entire SDSS footprint $\left(11,838.5 \pm 20.1 \mathrm{deg}^{2}\right)$, and the resulting QLF at the bright end at redshifts $2.8 \leqslant z \leqslant 4.5$. We also report the results of our spectroscopic identification campaign in the South Galactic Cap, the ELQS-S sample. We provide a brief introduction to the ELQS survey in Section 2. Subsequently, we discuss the ELQS-S observations and our data reduction in Section 3. The ELQS-S sample, including the discovery of 70 new quasars, is presented in Section 4, which leads to a discussion of the properties of the full ELQS quasar catalog (Section 5). Based on this catalog we calculate the QLF (Section 6) and discuss the implications of our results in Section 7. We summarize our findings in Section 8.

All magnitudes are displayed in the $\mathrm{AB}$ system (Oke \& Gunn 1983) and corrected for Galactic extinction (Schlafly \& Finkbeiner 2011) unless otherwise noted. We denote magnitudes not corrected for Galactic extinction only by $x$, where $x$ refers to the wavelength band in question, as opposed to extinction-corrected magnitudes $m_{\mathrm{x}}$. We adopt the standard flat $\Lambda$ CDM cosmology with $H_{0}=70 \mathrm{~km} \mathrm{~s}^{-1} \mathrm{Mpc}^{-1}, \Omega_{\mathrm{m}}=0.3$, and $\Omega_{\Lambda}=0.7$, which is generally consistent with recent measurements (Planck Collaboration et al. 2016).

\section{Introduction to the Extremely Luminous Quasar Survey (ELQS)}

The ELQS was designed to provide an accurate measure of the UV-bright type-I QLF at the bright end $\left(M_{1450}<-27\right)$ at intermediate redshifts $(2.8 \leqslant z \leqslant 4.5)$.

We apply a highly inclusive color cut in the $J-K-W 2$ plane $(K-W 2 \geqslant 1.8-0.848(J-K)$; Vega magnitudes) using photometry from the Two Micron All Sky Survey (2MASS, Skrutskie et al. 2006) and the Wide-field Infrared Survey Explorer mission (WISE, Wright et al. 2010). Using optical SDSS photometry along with WISE photometry we estimate photometric redshifts and further classify our candidates using random forests (Breiman 2001), a supervised machine learning technique. In both cases the random forest method is trained on a quasar sample built from the SDSS DR7 and DR12 quasar catalogs (Schneider et al. 2010; Pâris et al. 2017). The quasar selection is described in Paper I.

The ELQS covers the entirety of the SDSS footprint excluding the Galactic plane $\left(b<-20^{\circ}\right.$ or $\left.b>30^{\circ}\right)$, but including the SDSS strips at decl. $<0$. We have estimated the area coverage of our survey in Paper I using the Hierarchical Equal Area isoLatitude Pixelation (HEALPix; Górski et al. 2005). A description of the calculation process and the general parameters used can be found in Jiang et al. (2016). The effective area of the ELQS is $11,838.5 \pm 20.1 \mathrm{deg}^{2}$, of which $7601.2 \pm 7.2 \mathrm{deg}^{2}$ are part of the North Galactic Cap $\left(90^{\circ}<\right.$ R.A. $\left.<270^{\circ}\right)$ and $4237.3 \pm 12.9 \mathrm{deg}^{2}$ are part of the South Galactic Cap (R.A. $>270^{\circ}$ and R.A. $<90^{\circ}$ ).

We discuss our selection function in the second paper of this series, Paper II. For its calculation, we imposed our selection criteria, including the completeness limits of the photometric 
catalogs, on a sample of simulated quasar spectra uniformly distributed as a function of observed $i$-band magnitude and redshift. The resulting completeness reaches $>70 \%$ in the core region of our survey $\left(3.0 \lesssim z \lesssim 5.0 ; m_{i} \lesssim 17.5\right)$. We show the selection function of the ELQS survey as a function of redshift and apparent $i$-band magnitude in Figure 1, highlighting all newly discovered and already known quasars of the full ELQS sample in orange and blue, respectively.

We also presented the ELQS quasar sample in the North Galactic Cap $\left(90^{\circ} \leqslant\right.$ R.A. $\left.\leqslant 270^{\circ}\right)$ footprint $($ ELQS-N) in Paper II. This sample consists of 270 quasars at $m_{i} \leqslant 18.0$ and $z \geqslant 2.8$, of which 39 were newly identified as part of the ELQS survey.

Using 120 quasars from the ELQS-N sample, which adhere to the uniform photometric criteria of the 2MASS point source catalog (PSC) assumed by our calculation of selection function, we conducted a first analysis of the bright-end QLF. Single power-law fits to the data result in a steep value for the brightend slope of $\beta \approx-4$. We can further constrain the bright-end slope to $\beta<-2.94$ with $99 \%$ confidence. This result contrasts earlier QLF estimates at the same redshift (Fan et al. 2001; Richards et al. 2006), which find a generally flatter slope of $\beta \approx-2.5$.

The present work completes the ELQS survey with spectroscopic observations in the South Galactic Cap of the SDSS footprint. Our selection for this area resulted in a larger quasar candidate sample than for the ELQS-N, including many quasars that were not spectroscopically followed up by the original SDSS quasar survey. As a consequence, the ELQS-S sample presents a total of 70 newly discovered quasars, which allows for stronger statistical constraints on the QLF.

\subsection{ELQS Candidates in the Literature}

We have discussed the references for known quasars in the ELQS-N in some detail in Paper II (Section 2.2). Since all known quasars in the ELQS-S sample are from the same references, we will only present a summary below. For further details, please refer to Paper II.

The majority of known quasars in the ELQS-S sample were discovered by the SDSS (Abazajian et al. 2009), the BOSS, and eBOSS. The quasars are published in the SDSS DR7 (Schneider et al. 2010), DR12 (Pâris et al. 2017), and DR14 (Pâris et al. 2018) quasar catalogs.

In addition, we have matched against the Million Quasar Catalog (MQC; Flesch 2015) to identify known quasars that were not included in the SDSS quasar catalogs. The MQC is a compilation of quasars from a variety of different sources in the literature and includes quasar candidates as well. Only verified quasars were used in the cross-match between the catalog and our candidates.

We also match our quasar candidates against the most recent catalogs of the Large Sky Area Multi-Object Fibre Spectroscopic Telescope (LAMOST) quasar survey (Dong et al. 2018). The LAMOST quasar survey is part of the LAMOST Extragalactic Survey (Zhao et al. 2012), and quasars are selected using multicolor photometry color cuts as well as datamining algorithms. Three candidates of the ELQS-S sample are successfully matched to LAMOST quasars.

Furthermore, J. Yang et al. (2018, in preparation) are currently carrying out a spectroscopic survey similar to the ELQS. Their candidate selection consists of two samples that use optical and infrared color criteria presented in
Wu \& Jia (2010) and Wu et al. (2012). They aim to find bright quasars at $z \approx 2-3$ and at $z \geqslant 4$ missed by the SDSS/ BOSS/eBOSS quasar surveys and to test different quasar selection criteria for the upcoming LAMOST quasar survey. Their spectroscopic observations are conducted at the Lijiang telescope $(2.4 \mathrm{~m})$ and the Xinglong telescope $(2.16 \mathrm{~m})$.

We also discovered that one of our candidates, J215743.62 +233037.1, was part of the Hubble Space Telescope (HST) GO program $13013^{9}$ (PI: G. Worseck). While it was never published in a quasar catalog, it has been further studied by Zheng et al. (2015) and Schmidt et al. (2017). We decided to include it in our sample of newly discovered ELQS-S quasars so as to formally publish its classification, including an optical spectrum.

\section{Spectroscopic Observations and Data Reduction}

Exploratory observations for the ELQS started in 2015 and were designed to test a variety of selection criteria. As a result we discovered a range of quasars that are not included in the primary ELQS candidate catalog, which was finalized in 2016 September. We present their discovery spectra and their general properties in Appendix D.

Observations of the ELQS-S sample have been completed and 96 out of 97 candidates were observed with a range of different telescopes. These include the Vatican Advanced Technology Telescope (VATT), the MMT $6.5 \mathrm{~m}$ telescope, the 90 inch $(2.3 \mathrm{~m})$ Bok Telescope, the Nordic Optical Telescope (NOT), and the $(4.1 \mathrm{~m})$ Southern Astrophysical Research Telescope (SOAR). In this section we will detail the different instrumental setups and briefly describe the data reduction process.

\subsection{VATT Observations}

We have carried out the majority of our spectroscopic identifications with the VATTSpec spectrograph on the VATT. We used the 300 grooves $\mathrm{mm}^{-1}$ grating in first order blazed at $5000 \AA$. The spectra have a resolution of $R \sim 1000$ (1". 5 slit) and a coverage of $\sim 4000 \AA$ around our chosen central wavelength of $\sim 5775 \AA$.

The observations for the ELQS-S were conducted in multiple campaigns. Pilot observations started on 2015 October 8-12. The program continued on 2016 November 20-23 and December 18-20. In 2017 we finished the South Galactic Cap footprint during observations on November 7-12. Depending on the object and the conditions, the exposure times varied between 15 and 30 minutes.

\subsection{Bok Observations}

In fall 2016 we were awarded three nights on the Bok telescope. We used the Boller \& Chivens Spectrograph (B\&C spectrograph) with the 400 grooves $\mathrm{mm}^{-1}$ grating blazed at $4889 \AA$ in first order and the UV-36 blocking filter. The central wavelength was chosen to be $\sim 5250 \AA$, resulting in a coverage of $\approx 3655-6850 \AA$. The observations were conducted in 2016 on October 13-14 and November 15. The spectra were taken with the 2.15 slit, resulting in a resolution of $R \approx 750$. Depending on weather conditions and the apparent magnitude of the object, we used exposure times of $\sim 5-15$ minutes.

\footnotetext{
9 http://www.stsci.edu/hst/phase2-public/13013.pro
} 


\subsection{MMT Observations}

We have used the MMT Red Channel Spectrograph to carry out follow-up observations of our newly discovered quasars. For all observations we have used the MMT 270 grooves $\mathrm{mm}^{-1}$ and 300 grooves $\mathrm{mm}^{-1}$ gratings blazed at $1 \mathrm{st} / 7300 \AA$ and $1 \mathrm{st} / 4800 \AA$, respectively. With regard to the 270 grooves $\mathrm{mm}^{-1}$ grating we used central wavelengths of 6400,7000 , and $7150 \AA$. For the other grating we used central wavelengths of 5000, 5500, and $6083 \AA$. The 270 grooves $\mathrm{mm}^{-1}$ grating has an approximate coverage of $3705 \AA$, whereas the 300 grooves $\mathrm{mm}^{-1}$ grating has an approximate coverage of $3310 \AA$. We chose exposure times of 3-15 minutes per spectrum, depending on the object and conditions. Based on the seeing conditions, we have used either the 1 ." 25 or the 1 ." 5 slit, providing a resolution of $R \approx 300-400$ with both gratings. Observations were taken in 2017 on May 17-18, October 20-21, and November 16 and on 2018 January 20.

After the completion of the survey we noticed that the dim continuum lamp of the MMT Red Channel Spectrograph failed during our run on 2017 May 17-18, resulting in flat fields with very low signal-to-noise ratio for those two nights. We were able to re-reduce the spectra with the 300 grooves $\mathrm{mm}^{-1}$ grating and a central wavelength of $5560 \AA$ using flat fields from a different observing run. The flat fields with low signalto-noise ratio are still used for all spectra centered around $6083 \AA$, introducing additional noise. However, we do not expect any systematic biases because the detector of the spectrograph does not show strong variations in sensitivity along the spatial direction, and variations along the dispersion direction are indirectly taken care of by the standard calibration procedure.

\subsection{NOT Observations}

In 2017, some identification spectra were taken during the NOT summer schools (August 23-25, September 5-9). These observations were conducted with the Andalucia Faint Object Spectrograph and Camera (ALFOSC) using the 300 grooves $\mathrm{mm}^{-1}$ grism (\#4). The grism, centered around $5800 \AA$, offers a wavelength coverage of 3200-9600 . We used the blue blocking filter WG345 356_LP with a cut-on at $\sim 3560 \AA$. Given the above setup the spectra, taken with the 1 !" 0 and the 1 .' 3 slits, provide a resolution of $R \approx 360$ and $R \approx 280$, respectively. Exposure times varied between 2.5 and 5 minutes, depending on atmospheric transparency and apparent target magnitude.

\subsection{SOAR}

In addition, we observed quasar candidates with the Goodman High Throughput Spectrograph (Goodman HTS) on the SOAR $(4.1 \mathrm{~m})$. These observations were carried out on 2017 October 6-10 and 2018 January 22-24. We used the 400 grooves $\mathrm{mm}^{-1}$ grating with central wavelengths of 6000 and $7300 \AA$. The spectra have a spectral coverage of $\sim 4000-8000 \AA$ and $\sim 5300-9300 \AA$, respectively. The first setup used the GG-385 blocking filter, whereas the second one used the GG-495 blocking filter. We used the red camera in $2 \times 2$ spectral mode for all observations. Dependent on the weather conditions, we chose the 1 !" 0 or 1 ". 2 slit, resulting in spectral resolutions of $R \approx 830$ and $R \approx 690$, respectively. Exposure times varied between 3 and 15 minutes depending on the target magnitude as well as the atmospheric transparency.

\subsection{Data Reduction}

The data were reduced using the standard long-slit reduction methods within the IRAF software package (Tody 1986, 1993). This includes bias subtraction, flat field corrections, and sky subtractions using polynomial background fits along the slit direction. The last task was carried out using the apall routine. All observations since 2016 October were reduced using optimal extraction (weights $=$ variance) and cosmic ray reduction within the apall routine. Our observations resulted in spectra with low to medium signal-to-noise ratio. In all cases quasars were easily classified by their broad emission lines. Furthermore we have used internal lamps for wavelength calibration and observed at least one spectrophotometric standard star per night. Because of changing weather conditions our absolute flux calibration may not reliable. Therefore the fluxes were scaled to match the SDSS $r$-band magnitudes. The spectra have not been corrected for telluric absorption features.

\section{The ELQS-S Quasar Sample}

The ELQS-S sample covers the Southern Galactic Cap of the SDSS footprint (R.A. $>270^{\circ}$ or R.A. $<90^{\circ}$ ). We have selected 219 primary candidates in this area of the ELQS. Of these, 50 candidates were discarded during visual inspection of the photometry. In most of these cases the objects were strongly blended in the WISE bands or showed photometric artifacts (bright trails identified as the source). Of the remaining 169 primary candidates 72 are known in the literature. These include 67 objects at $z \geqslant 2.8$ (DR14Q: 35 objects, MQC: 16, Yang et al.: 13, LAMOST DR2/3: 3) as well as five objects at $z<2.8$ (MQC: 3 objects, DR14Q: 1, SDSS spectrum: 1). An overview of the ELQS primary candidate sample is given in Table 1.

We obtained optical spectroscopy for 96 out of the remaining 97 unknown candidates and discovered 70 new quasars at $z \geqslant 2.8$ and four at $z<2.8$. The majority of our 22 contaminants in the spectroscopic sample are stars (21), predominantly K-dwarfs (13), which have optical colors similar to the quasars in our targeted redshift range.

In total the ELQS-S catalog includes 137 quasars at $z \geqslant 2.8$ :

1. 70 newly identified quasars.

2. 35 quasars from the DR14Q.

3. 16 quasars from MQC.

4. 13 quasars from J. Yang et al. (2018, in preparation).

5. 3 quasars from LAMOST DR2/3.

Excluding the 50 primary candidates with unreliable photometry, we have successfully selected 137 quasars at $z \geqslant 2.8$ out of 169 candidates. Therefore the ELQS-S sample has a selection efficiency of $\sim 80 \%$, consistent with the ELQS$\mathrm{N}$ sample.

We show the distribution of all good primary candidates in the ELQS-S sample as a function of dereddened SDSS $i$-band magnitude in Figure 2. Known quasars from the literature are shown in blue, while new ELQS-S quasars at $z \geqslant 2.8$ and $z<2.8$ are displayed in red and green, respectively. All objects that were spectroscopically identified not to be quasars are colored orange. In addition, two objects could not be identified. One of them was not observed and the spectrum of the other one had too low a signal-to-noise ratio to allow for a reliable classification. These two objects are shown in gray.

The figure shows a significant dip in quasar candidates around $m_{i} \sim 17.75$. This can be explained by our selection 


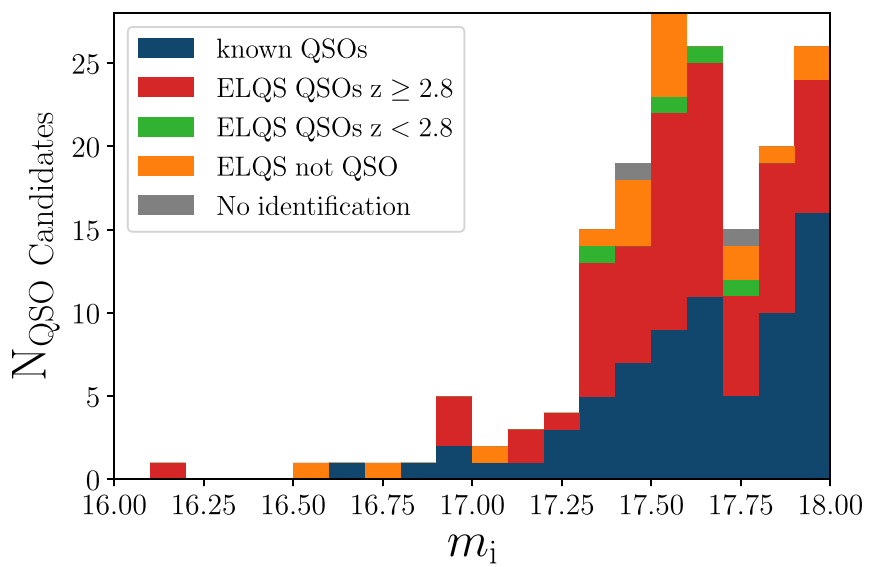

Figure 2. We present the distribution of all good primary ELQS-S candidates as a function of their apparent SDSS $i$-band magnitude. Quasars known from the literature are colored blue. Red and green colors highlight the newly discovered quasars with $z \geqslant 2.8$ and $z<2.8$, respectively. Candidates that have been identified not to be quasars are shown in orange, while objects that could not be identified or were not observed are shown in gray.

function (Figure 1). Our estimated selection completeness drops below $50 \%$ at magnitudes fainter than $m_{i}=17.5$. That the number of quasar candidates rises again in the two faintest magnitude bins is due to the intrinsic number of quasars rising at these magnitudes. The same phenomenon is evident in our ELQS-N candidates (see Figure 1 in Paper II). Here, the number of quasar candidates stagnates around $m_{i} \sim 17.6-17.8$ and then increases strongly beyond $m_{i} \gtrsim 17.8$.

The 70 discovery spectra of our newly identified quasars are displayed in Figure 9. The spectra are ordered in redshift, beginning with the lowest at $z=2.82$. According to the spectroscopic redshift we highlight the positions of the broad Ly $\alpha$, C IV, and Si IV emission lines with blue, orange, and red bars at the top of each spectrum. The redshift and the designation of the object are shown in either the top right or top left corner of each spectrum. In a few cases the flux correction introduced a rising continuum at the blue end, which is likely due to insufficient signal at the bluest wavelengths. For example, J012535.83+401425.5 and J235330-050817.8 are affected by this problem.

Spectroscopic redshifts are measured by visually matching a quasar template spectrum (Vanden Berk et al. 2001) to the observed spectra. We estimate that the uncertainty in redshift introduced by this method is $\Delta z \approx 0.02$, which is accurate enough for the calculation of the QLF.

$\mathrm{K}$-corrections are calculated in the same fashion as for the ELQS-N sample. We have used the sample of simulated quasar spectra (see Section 5.1 in Paper II) to derive a K-correction term as a function of redshift and magnitude to calculate the monochromatic magnitude at rest-frame $1450 \AA$ from the SDSS $i$-band magnitude. The simulated quasar spectra were calculated on a narrow grid in redshift and absolute magnitude, and K-corrections are calculated for each grid cell. This grid is then interpolated to retrieve individual K-corrections for each quasar in our sample.

Figure 3 shows the distribution of all quasars in the ELQS as a function of $M_{1450}$ and redshift. Known quasars identified by SDSS spectroscopy or included in the SDSS DR7 and DR14 quasar catalogs are shown as blue dots and labeled "SDSS." Other known quasars, which are part of the MQC or the quasar sample of J. Yang et al. (2018, in preparation) are marked with green triangles. ELQS quasars are highlighted as red diamonds, where solid diamonds refer to the new ELQS-S sample and open diamonds to the ELQS-N sample of Paper II. The histograms show the binned distribution as a function of their respective axis. The three green stars are the well known quasar lenses Q1208+1011 ( $z=3.8)$ (Bahcall et al. 1992; Magain et al. 1992), B1422+231B ( $z=3.62)$ (Patnaik et al. 1992), and APM $08279+5255(z=3.91)$ (Ibata et al. 1999) and were selected as part of ELQS-N.

For all newly discovered quasars in the ELQS-S sample we provide additional information in Table 2. This includes the position in equatorial coordinates, SDSS apparent $i$-band magnitude, the absolute magnitude at $1450 \AA$, near- and farUV magnitudes from Galaxy Evolution Explorer (GALEX) GR6/7, a flag indicating visual broad absorption line (BAL) quasar classification, the determined spectroscopic redshift, and further notes.

\section{The Full ELQS Quasar Catalog}

The full ELQS quasar catalog comprises 407 objects, of which 109 are newly identified. The previously published ELQS-N catalog (Paper II), covering only the North Galactic Cap of the SDSS footprint, included 270 quasars (new: 39, known: 231). With this work we add the Southern Galactic Cap footprint of the ELQS, identifying 70 new quasars and effectively more than doubling the number of selected known quasars in this area. The selection criteria and selection function are identical to those in Paper II. Across the entire ELQS we selected 509 primary quasar candidates, of which 407 were identified to be quasars at $z \geqslant 2.8$, resulting in an overall selection efficiency of $\sim 80 \%$. The SDSS quasars make up only $\sim 60 \%$ of the ELQS sample and $80 \%$ of all previously known quasars in the SDSS footprint, if all known quasars from the literature are included. This demonstrates that our selection is more inclusive than the SDSS quasar selection, allowing us to recover an additional 50 quasars known in the literature.

We matched the full ELQS sample against known quasar lenses. This includes a list of known quasar lenses in the NASA/IPAC Extragalactic Database (NED), the CfA-Arizona Space Telescope Lens Survey of gravitational lenses (CASTLES, C. S. Kochanek, E. E. Falco, C. Impey, J. Lehar, B. McLeod, H.-W. Rix), ${ }^{10}$ and the SDSS Quasar Lens Search (SLQS, Inada et al. 2012). The three returned matches are the well known quasar lenses Q1208+1011 $(z=3.8)$ (Bahcall et al. 1992; Magain et al. 1992), B1422+231B $(z=3.62)$ (Patnaik et al. 1992), and APM 08279+5255 $(z=3.91)$ (Ibata et al. 1999), which were already included in the ELQS-N sample. These are highlighted as green stars in Figure 3.

One of our candidates, J035047.55+143908.2, remains unobserved, and the spectrum of another one, J025204.49 +201407.9 , has too low a signal-to-noise ratio to allow for an unambiguous classification. Therefore the ELQS is $99.6 \%$ spectroscopically complete.

\subsection{Matches to FIRST and the Radio-loud Fraction (RLF)}

We match the full ELQS sample to sources in the VLA Faint Images of the Radio Sky at Twenty Centimeters (FIRST) catalog (Becker et al. 1995) in an aperture of 3!"0. We obtain

\footnotetext{
${ }^{10}$ https://www.cfa.harvard.edu/castles/
} 
Table 1

ELQS Primary Candidate Sample

\begin{tabular}{|c|c|c|c|}
\hline $\begin{array}{l}\text { Primary Candidates } \\
\left(m_{i} \leqslant 18.0 \text { and } z_{\text {reg }} \geqslant 2.8\right)\end{array}$ & Full Area & $\begin{array}{c}\text { ELQS-N } \\
\left(90^{\circ}<\text { R.A. }<270^{\circ}\right)\end{array}$ & $\begin{array}{c}\text { ELQS-S } \\
\left(\text { R.A. }>270^{\circ} \text { or R.A. }<90^{\circ}\right)\end{array}$ \\
\hline Total selected primary candidates & 594 & 375 & 219 \\
\hline Good primary candidates (excluding bad photometry) & 509 & 340 & 169 \\
\hline Good primary candidates in the literature & 324 & 252 & 72 \\
\hline Good primary candidates observed & 184 & 88 & 96 \\
\hline Good primary candidates to observe & 1 & 0 & 1 \\
\hline Good primary candidates in the literature at $z>2.8$ & 298 & 231 & 67 \\
\hline Good primary candidates observed and identified as $z>2.8$ QSOs & 109 & 39 & 70 \\
\hline
\end{tabular}

measured $1.4 \mathrm{GHz}$ flux densities for a total of 37 matches (ELQS-N: 34, ELQS-S: 3). All three matches in ELQS-S are to quasars already known in the literature. The full ELQS catalog (Appendix A) includes information on the match distance to the FIRST source, its $1.4 \mathrm{GHz}$ peak and integrated flux density, as well as the rms error on the integrated flux density.

Since the FIRST footprint has been chosen to coincide with the SDSS North Galactic Cap footprint, we can estimate the RLF of our ELQS-N quasar sample. This allows us to test whether the ELQS quasar sample has similar or different radio properties to other surveys. In Paper II we simply counted all sources with $1.4 \mathrm{GHz}$ peak flux detections, which resulted in an RLF of $\approx 12.6 \%$.

Jiang et al. (2007) have analyzed the RLF for a large sample of SDSS quasars at $z=0-5$ and $-30 \leqslant M_{i}<-22$. They define a radio-loud quasar based on its $R$ parameter, the ratio of the flux density at $6 \mathrm{~cm}(5 \mathrm{GHz})$ to flux density at $2500 \AA$ in the rest-frame,

$$
R=f_{6 \mathrm{~cm}} / f_{2500} .
$$

In their analysis they calculate $f_{6} \mathrm{~cm}$ from the $1.4 \mathrm{GHz}$ integrated flux density (if detected) by assuming a power-law slope of $\alpha=-0.5$. They further obtain the observed flux density $f_{2500}$ at rest-frame $2500 \AA$ by fitting a model spectrum to the SDSS broadband photometry. Quasars are then counted as radio-loud for all values of $R \geqslant 10$. They discovered that the RLF changes as a function of redshift and absolute magnitude and is well fit by

$$
\log _{10}\left(\frac{\mathrm{RLF}}{1-\mathrm{RLF}}\right)=b_{0}+b_{z}(1+z)+b_{M}\left(M_{2500}+26\right),
$$

where $b_{0}=-0.132, b_{z}=-2.052$, and $b_{M}=-0.183$.

We revisit our analysis of the ELQS-N RLF by using the same criterion for radio-loud quasars as Jiang et al. (2008). In our case we calculate the observed flux density $f_{2500}$ using the $\mathrm{K}$-correction estimated from our sample of simulated quasars (Paper II, Section 5.1). Our K-correction is not only based on a quasar continuum model, but also includes contributions from the broad quasar emission lines. The rest-frame flux density at $6 \mathrm{~cm}, f_{6 \mathrm{~cm}}$, is derived identically to Jiang et al. (2007), by assuming a power-law slope of $\alpha=-0.5$ for the K-correction.

We calculate the RLF for three different subsamples of the ELQS-N catalog restricted by $m_{i} \leqslant 17.0,17.5$, and 18.0. In all cases we calculate median absolute magnitudes and redshifts as input into the relation found by Jiang et al. (2007). We compare results from the ELQS sample in Table 3 with the RLF calculated using the best-fit relation. Uncertainties on our measured RLF are derived assuming a Poisson distribution $(\sigma=\sqrt{N})$.

Compared to our previous estimate of $\mathrm{RLF}=12.6 \%$ $\left(m_{i} \leqslant 18.0\right)$ in Paper II, where we only counted quasars with radio detections, our more rigorous RLF estimates agree with the values derived from the relation of Jiang et al. (2007) at the $1.5 \sigma$ level. This largely confirms that the ELQS quasar sample has similar radio properties to previous SDSS surveys.

\subsection{Matches to GALEX, ROSAT $2 R X S$, and XMMSL2}

We have cross-matched the full ELQS sample with the GALEX GR6/7 Data Release (Martin et al. 2005). Matches are evaluated within an aperture of 2 !" 0 , which corresponds to the GALEX position accuracy. For all matches we have obtained the available photometry in the near- and far-UV bands at 1750-2750 $\AA$ and 1350-1750 $\AA$, respectively. The near- and far-UV magnitudes for the ELQS-S sample are also displayed in Table 2.

We obtained 55 GALEX matches to the full ELQS sample (ELQS-N: 38, ELQS-S: 17). Of these matches 52 (ELQS-N: 37, ELQS-S: 15) are detected in the near-UV band and 19 (ELQS-N: 10, ELQS-S: 9) in the far-UV band. A subset of 16 (ELQS-N: 9, ELQS-S: 7) sources were detected in both bands. We have discovered 109 (ELQS-N: 39, ELQS-S: 70) new quasars with ELQS, of which 14 (ELQS-N: 8, ELQS-S: 6) have GALEX counterparts in either or both photometric bands.

There are three new ELQS-S quasars (J004021.734033451.36, J215558.301+022856.12, and J215743.626 +233037.34 ), which are detected in both near- and far-UV GALEX bands, while two objects only have far-UV photometry and one has only near-UV photometry available.

The detection of high-redshift quasars in near- and far-UV bands in the observed frame suggests that their flux has not been fully absorbed by intervening neutral hydrogen along the line of sight. Thus, these objects are prime targets in which to study the helium reionization of the universe (Worseck \& Prochaska 2011; Worseck et al. 2016).

The rate of UV detections in the full ELQS sample $(55 / 407 \approx 13.5 \%)$ is very similar to the rate of UV detections of our newly identified ELQS quasars $(14 / 109 \approx 13 \%)$. In Paper II we discussed the rate of UV detections in the ELQS-N sample and found that a large fraction of newly identified quasars $(8 / 39 \approx 20 \%)$ have UV detections compared to the overall ELQS-N sample $(38 / 270 \approx 14 \%)$.

Worseck \& Prochaska (2011) found that the SDSS quasar sample preferentially selects quasars with intervening H I Lyman-limit systems. In the case of the North Galactic Cap, 


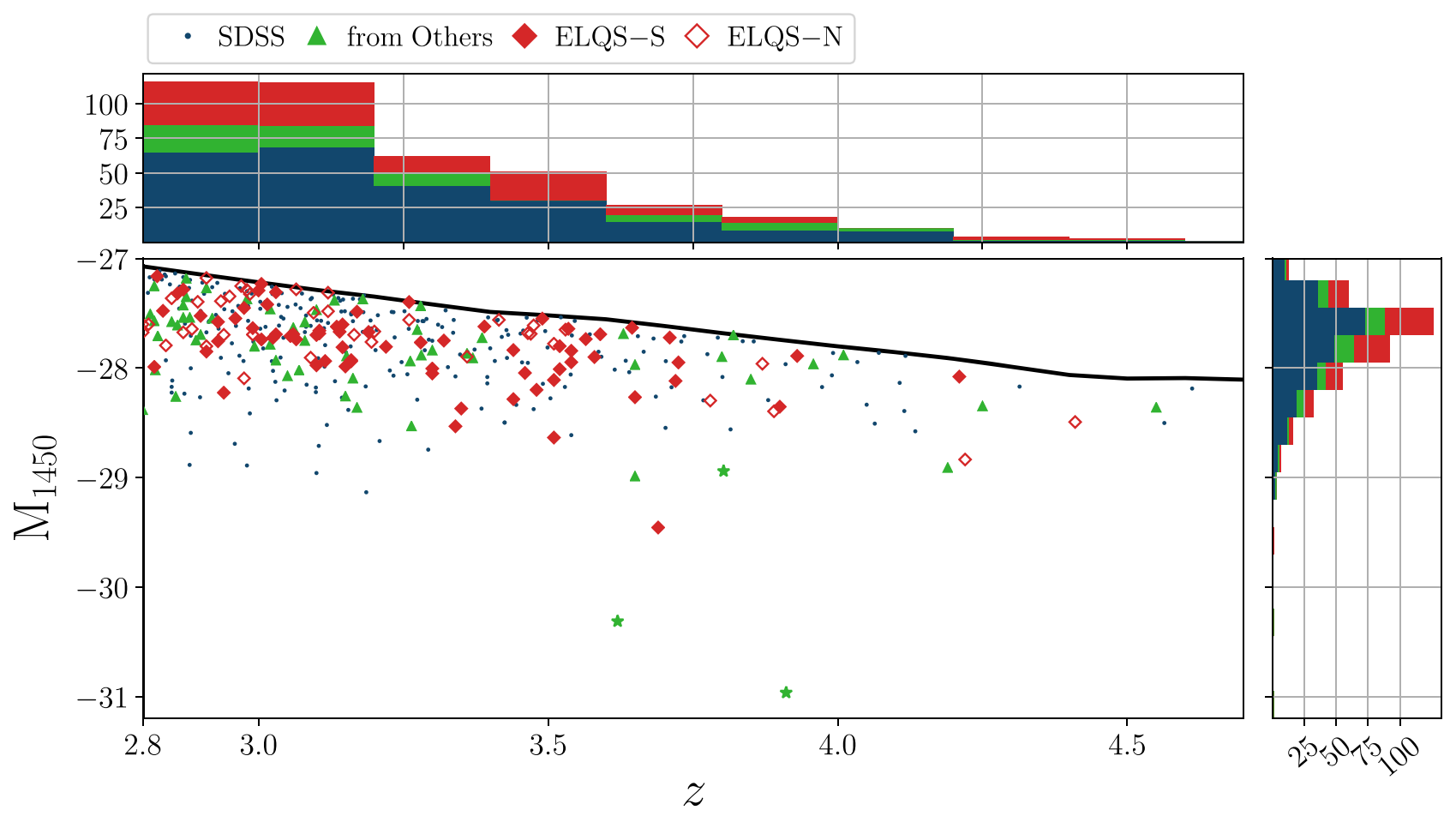

Figure 3. The distribution of all quasars in the full ELQS sample as a function of absolute $1450 \AA$ magnitude $\left(M_{1450}\right)$ and redshift $(z)$. Quasars identified with SDSS spectroscopy or as part of the SDSS DR7Q and DR14Q are shown as blue dots and labeled "SDSS." We also include a range of quasars that were not (re)discovered by SDSS with identifications from the Million Quasar Catalog (MQC) or the quasar sample of J. Yang et al. (2018, in preparation). These objects are depicted in green (triangles, stars). Newly identified ELQS quasars are shown as red diamonds. Solid diamonds refer to the ELQS-S sample, whereas open diamonds highlight quasars of the ELQS-N sample presented in Paper II. We also show the distribution of all quasars in histograms along both axes. The three green stars are the well known quasar lenses Q1208+1011, B1422+231B, and APM 08279+5255.

where SDSS spectroscopic follow-up is completed, we would therefore expect the SDSS to have missed a larger fraction of quasars with UV detections. Assuming that our selection does not carry the same bias, we would naturally find a larger fraction of UV detections among our new ELQS-N quasars. However, SDSS spectroscopic follow-up has not been completed in the South Galactic Cap footprint, leaving a larger and more unbiased fraction of quasars undiscovered. This could explain the UV detection rates of our newly identified quasars compared to the full ELQS sample.

We further cross-matched all quasars in the full ELQS catalog with pre-matched AllWISE counterparts to X-ray detections (Salvato et al. 2018) from the ROSAT (Truemper 1982) reprocessed 2RXS catalog (Boller et al. 2016) and the XMM Newton Slew 2 Survey (XMMSL2). These catalogs contain 106,573 counterparts to $0.1-2.4 \mathrm{keV} 2 \mathrm{RXS}$ sources as well as 17,665 counterparts to $0.2-12 \mathrm{keV}$ XMMSL2 sources. We matched the AllWISE positions of the sources in our sample to the AllWISE positions of the counterparts in a $6^{\prime \prime}$ aperture.

While we find no matches to the XMMSL2 counterparts, we recover 11 sources that have ROSAT 2RXS detections. All of them are already known quasars in the literature. The ROSAT 2RXS fluxes are included in the full ELQS quasar catalog (see Appendix A).

\subsection{BAL Quasar Fraction}

We revisit our previous estimate of the fraction of BAL quasars of Paper II (Section 4.2) with the full ELQS sample. While a thorough quantitative analysis of the BAL quasar fraction would require the calculation of the balnicity index
(BI) (Weymann et al. 1991) or the absorption index (Hall et al. 2002) from the spectral data, this is beyond the scope of this work. Traditionally BAL quasars are classified by BI $>0$. However, we limit ourselves to a qualitative analysis of the BAL quasar fraction by visually classifying all ELQS quasars as BAL quasars and non-BAL quasars. Based on this classification we roughly estimate the BAL fraction of the ELQS quasar sample.

As in our previous analysis of the ELQS-N sample, we crossmatch the full ELQS catalog to the SDSS DR12 quasar catalog (Pâris et al. 2017) and retrieve information on visual BAL quasar classifications (BAL_FLAG_VI = 1). The DR12Q BAL flag provides information on 212 (ELQS-N: 190, ELQS-S: 22) of our 407 quasars, of which 42 (ELQS-N: 40, ELQS-S: 2) are flagged as BAL quasars.

We visually inspect the spectra of all remaining objects, where available, or use previous classifications from the literature to determine their nature. Of all newly identified ELQS quasars 17 display BAL features. This includes six quasars of the ELQS-N sample and 11 new quasars of the ELQS-S (J001311.09+205342.8, J003901.10-214429.1, $\mathrm{J} 005248.64+215325.7, \mathrm{~J} 013223.20+184155.6, \mathrm{~J} 013807.12$ +172414.8 , J020256.07+312620.8, J031307.14+024515.3, J210827.25-030847.8，J224610.79-064953.7，J231334.60101152.3, J233117.24-054020.8). A total of seven ELQS quasars do not have sufficient signal-to-noise ratio or wavelength coverage in their discovery spectra to allow for unambiguous classification. Including all quasars from the literature, we could identify 79 BALs out of a sample of 384 ELQS quasars, resulting in a visual BAL quasar fraction of $\sim 21 \%$. For a total of 23 quasars we were not able to 
Table 2

Newly Discovered Quasars at $z \geqslant 2.8$ in the ELQS-S Sample

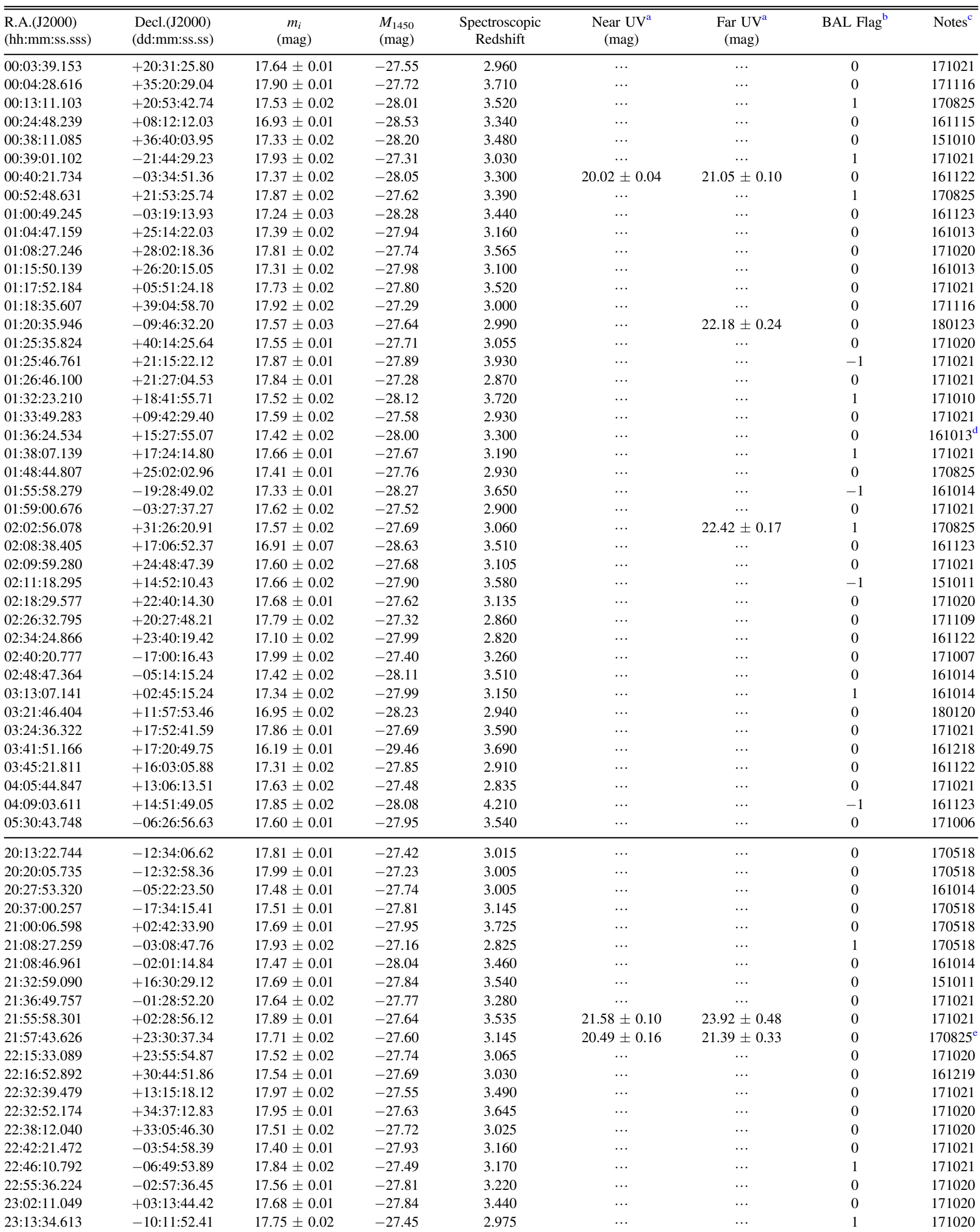


Table 2

(Continued)

\begin{tabular}{|c|c|c|c|c|c|c|c|c|}
\hline $\begin{array}{l}\text { R.A.(J2000) } \\
\text { (hh:mm:ss.sss) }\end{array}$ & $\begin{array}{l}\text { Decl.(J2000) } \\
\text { (dd:mm:ss.ss) }\end{array}$ & $\begin{array}{c}m_{i} \\
(\mathrm{mag})\end{array}$ & $\begin{array}{l}M_{1450} \\
(\mathrm{mag})\end{array}$ & $\begin{array}{l}\text { Spectroscopic } \\
\text { Redshift }\end{array}$ & $\begin{array}{l}\text { Near UV } \\
\text { (mag) }\end{array}$ & $\begin{array}{c}\text { Far UV } \\
(\mathrm{mag})\end{array}$ & BAL Flag ${ }^{\mathrm{b}}$ & Notes $^{c}$ \\
\hline 23:22:33.545 & $+17: 53: 09.61$ & $17.69 \pm 0.01$ & -27.75 & 3.320 & $\cdots$ & $\cdots$ & 0 & 170825 \\
\hline $23: 24: 52.615$ & $+18: 24: 16.53$ & $17.10 \pm 0.02$ & -28.37 & 3.350 & $\cdots$ & $\ldots$ & 0 & 171110 \\
\hline 23:33:20.540 & $+12: 20: 22.14$ & $17.59 \pm 0.01$ & -27.70 & 3.100 & $\cdots$ & $\cdots$ & 0 & $170825^{\mathrm{d}}$ \\
\hline 23:38:39.729 & $+29: 24: 21.00$ & $17.40 \pm 0.02$ & -28.35 & 3.900 & $\cdots$ & $\cdots$ & -1 & 171116 \\
\hline 23:53:08.773 & $+37: 44: 59.07$ & $17.36 \pm 0.02$ & -27.94 & 3.115 & $\ldots$ & $\ldots$ & 0 & 161013 \\
\hline
\end{tabular}

Notes.

${ }^{a}$ The near- and far-UV magnitudes were obtained from cross-matches within 2!. 0 to the GALEX GR6/7 data release.

${ }^{\mathrm{b}}$ Visual qualitative BAL identification flag: $1=\mathrm{BAL} ; 0=$ no BAL; $-1=$ insufficient wavelength coverage or inconclusive archival data.

${ }^{c}$ This column shows the observation date (YYMMDD) and provides further information on individual objects.

d These objects were also independently discovered by Yang et al.

${ }^{\mathrm{e}}$ See also HST GO Proposal 13013 (PI: Gabor Worseck), Zheng et al. (2015), and Schmidt et al. (2017).

Table 3

Radio-loud Fraction of the ELQS-N Sample Compared to the Relation of Jiang et al. (2007)

\begin{tabular}{cccc}
\hline \hline & $m_{i} \leqslant 17.0$ & $m_{i} \leqslant 17.5$ & $m_{i} \leqslant 18.0$ \\
\hline$N_{\text {ELQS }}$ & 22 & 92 & 270 \\
$z_{\text {median }}$ & 3.30 & 3.42 & 3.12 \\
$M_{2500, \text { median }}$ & -28.75 & -28.3 & -27.8 \\
$N_{\text {ELQS }}(R>10)$ & 2 & 10 & 25 \\
\hline $\mathrm{RLF}_{\text {ELQS }}$ & $(9.1 \pm 4.5) \%$ & $(10.9 \pm 2.3) \%$ & $(9.3 \pm 1.2) \%$ \\
$\mathrm{RLF}_{\text {Jiang 2007 }}$ & $10.6 \%$ & $8.4 \%$ & $8.0 \%$ \\
\hline
\end{tabular}

determine a classification, due to the lack or quality of the identification spectra.

With regard to the new ELQS-S sample, presented in this work, we have identified 22 out of 120 quasars as showing BALs. Therefore the ELQS-S BAL quasar fraction is $\sim 18 \%$, about $4 \%$ lower than in the ELQS-N sample.

The observed ELQS BAL quasar fraction of $\sim 21 \%$ remains high compared to previous studies in the literature (see discussion in Section 4.2 of Paper II). While Trump et al. (2006) find an observed traditional BAL fraction of $\sim 10 \%$ $(z=1.7-4.38)$ in the SDSS DR3 quasar catalog, quasar samples selected from near-infrared/infrared photometry have resulted in larger fractions of BAL quasars $(\sim 17.5 \%$, Maddox et al. 2008).

However, it remains unclear whether our infrared-based quasar selection (in the observed frame) (Dai et al. 2008; Maddox et al. 2008), our sampled redshift range, or our focus on the luminous end of the quasar distribution biases our quasar sample toward a high observed BAL fraction. In the future it would be interesting to conduct a more detailed analysis of the balnicity and absorption index for a large mid-infrared-selected type-I quasar sample to calculate the BAL fraction as a function of redshift and absolute magnitude. Different optical quasar selection criteria applied to the mid-infrared-selected quasar sample could then quantify the optical selection bias.

\section{The ELQS Quasar Luminosity Function}

Using the full ELQS sample, we re-evaluate our measurements of the QLF presented in Paper II, Section 6. We calculate the binned QLF, evaluate number density and redshift evolution using a non-parametric approach, and finally use a maximum likelihood method to constrain parameters for a single power-law and a broken double power-law fit to the data. Unfortunately, we have to limit our quasar sample to the stringent photometric criteria of the 2MASS PSC that we adopted for our completeness calculation (Paper II, Section 5.2.1). We therefore have to exclude 241 quasars of our full ELQS sample, leaving 166 quasars to determine the bright-end slope of the QLF. Figure 1 highlights all ELQS quasars that are included as part of the QLF sample on top of a map of the ELQS selection function. However, the majority of excluded quasars are at the faint end of the ELQS sample. Therefore this does not reduce the number of objects vital for analysis of the bright-end slope. Out of these 166 quasars, 38 are newly discovered and another 24 were not (re)discovered by SDSS. Therefore this sample includes 62 quasars that are not part of the SDSS quasar samples, a fraction of $37.35 \%$.

\subsection{The Binned $Q L F$}

We evaluate the binned QLF over the entire ELQS footprint $\left(11,838.5 \pm 20.1 \mathrm{deg}^{2}\right.$, see Paper I) using the $1 / V_{\mathrm{a}}$ method (Schmidt 1968; Avni \& Bahcall 1980) with the modification of Page \& Carrera (2000). We construct four redshift and five magnitude bins analogously to Paper II. The bin edges are $z=2.8,3.0,3.5,4.0,4.5$ and $M_{1450}=$ $-29.1,-28.7,-28.3,-28,-27.7,-27.5$. We use the previously determined selection function (Paper II, Section 5) to correct for incompleteness.

Figure 4 shows the binned QLF for the full ELQS sample (red data points, see also Table 4) compared to our previous estimate (ELQS-N in gray) and two other optical QLFs determined on the SDSS DR3 (orange, Richards et al. 2006) and DR9 (blue, Ross et al. 2013) quasar samples. We have converted the data of Richards et al. (2006) and Ross et al. (2013), given in absolute $i$-band magnitudes $M_{i}[z=2]$ continuum K-corrected to a redshift of $z=2$, to absolute magnitudes at $1450 \AA\left(M_{1450}\right)$, assuming a spectral index of $\alpha_{\nu}=-0.5\left(f_{\nu} \propto \nu^{\alpha}\right)$.

The binned QLF of Ross et al. (2013) has been chosen to exactly match our lower two redshift bins. While the binned QLF of Richards et al. (2006) matches our three higher redshift 


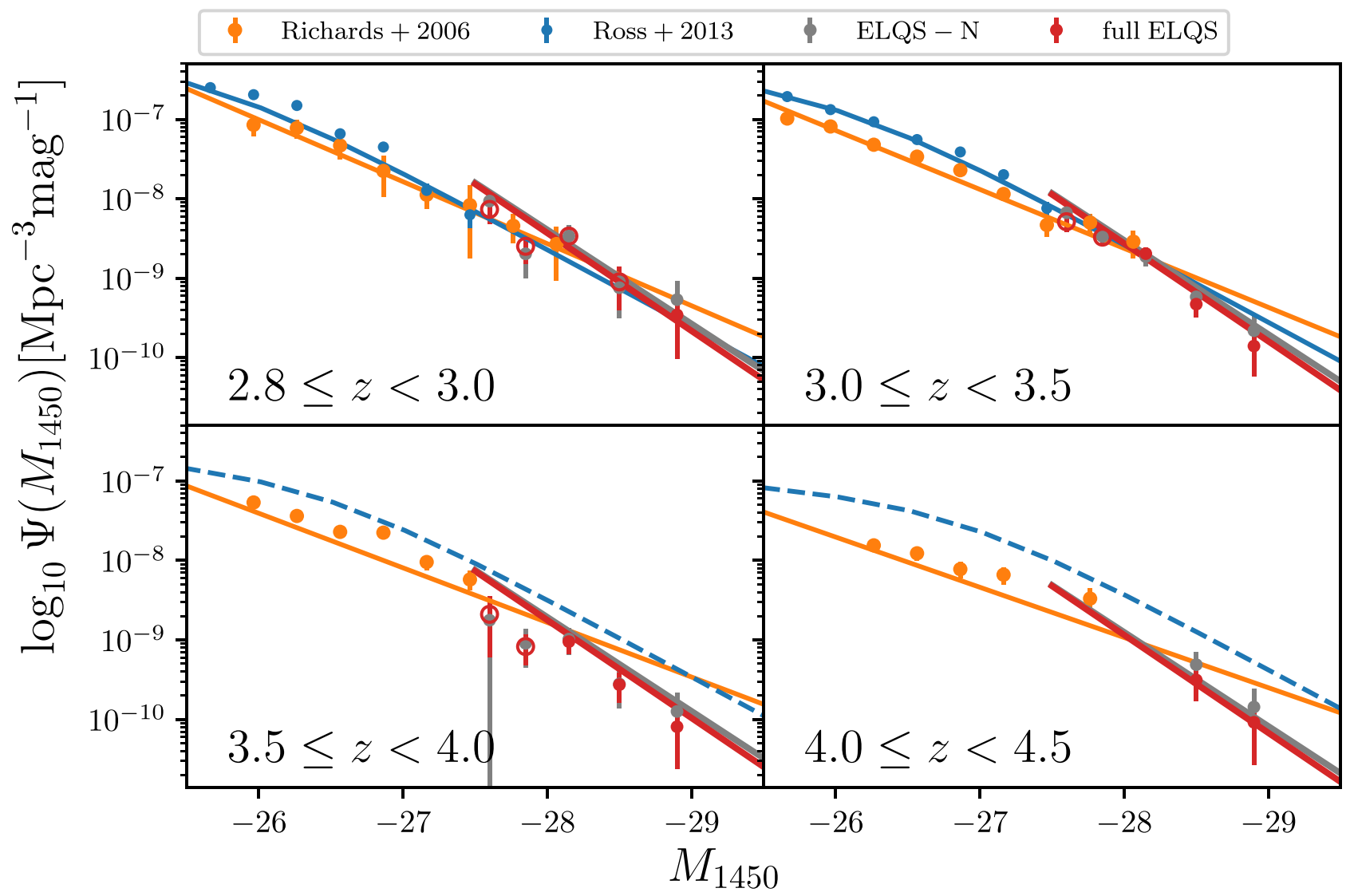

Figure 4. The QLF of UV-bright type-I quasars as a function of absolute magnitude, $M_{1450}$, in four redshift bins. Previous results on the QLF are from the original SDSS DR3 (Richards et al. 2006, orange) and the BOSS DR9 (Ross et al. 2013, blue). The full binned ELQS is shown in red. Red open circles denote the data points that either are derived from unfilled bins or have an average completeness below $50 \%\left(N_{\text {corr }} / N \geqslant 2\right)$. We show an earlier estimate of the QLF based on the ELQS-N sample in gray for comparison. The $1 \sigma$ error bars show the purely statistical error due to the number of quasars per bin. The lines show parametric fits of the QLF to quasar distributions, where dashed lines indicate an extrapolation of the QLF prescription to higher redshifts. The red lines are from the maximum likelihood fit to the full ELQS sample (Section 6.3, Table 5 first row). The orange and blue lines correspond to the parametric fits to the QLF of Richards et al. (2006, second row in their Table 7) and Ross et al. (2013, PLE (first row) + LEDE(S82) in their Table 8), respectively.

bins, their lowest redshift bin covers $z=2.6-3.0$ rather than our coverage of $z=2.8-3.0$.

The full binned ELQS QLF is shown with filled red circles. Data points in bins that are not fully filled or where the completeness is below $50 \%\left(N_{\text {corr }} / N \geqslant 2\right)$ are displayed with open red circles. These data points are prone to substantial systematic biases due to our selection function, and we caution against their overinterpretation. The error bars on the binned QLF only reflect statistical uncertainties based on the detected number of quasars per bin. For comparison we show the binned QLF of the ELQS-N sample (Paper II) in gray.

Figure 4 also displays the best fits to SDSS DR3, SDSS DR9, and ELQS quasar samples as solid lines. The color scheme follows the binned QLF. While the SDSS DR3 quasar sample (Richards et al. 2006) has been fit by a single power law, the SDSS DR9 quasar sample (Ross et al. 2013) extending to lower luminosities used a broken DPL parameterization. The ELQS sample, focused on the bright quasars, does not sample beyond the projected break of the broken DPL. Therefore, our sample can be described with a single power law. The values for the full ELQS and the ELQS-N fit are taken from Table 5 (first row) and Paper II (Table 5, first row), respectively. In all cases the fits are evaluated in the centers of the four redshift bins. We extrapolated the best fit of the SDSS DR9 QLF (Ross et al. 2013, see their Table 8: PLE (first row) + LEDE(S82)) beyond $z=3.5$, highlighted by the dashed line, to allow for a visual comparison in all redshift bins.

The ELQS survey allows us to extend the measurement of the QLF by one magnitude at the bright end up to $M_{1450} \approx-29$. In the brightest bin $\left(M_{1450} \approx-29.1\right.$ to -28.7$)$ the QLF reaches values around $10^{-10} \mathrm{Mpc}^{-3} \mathrm{mag}^{-1}$ at $z \geqslant 3.0$. The data points of our binned QLF demonstrate that the brightend slope is generally steeper, as anticipated by the extrapolation of the QLF fits from Richards et al. (2006) and Ross et al. (2013) toward the brightest magnitudes. This trend is especially clear in the full ELQS sample, which results in an even steeper slope than our previous measurement based on the ELQS-N sample.

\subsection{The Differential Marginal Luminosity Function}

The QLF is generally a function of luminosity and redshift. Binned approaches need to divide the sample into subsamples and estimate the quasar number density per magnitude in each bin to calculate the QLF. If we can assume that the redshift and luminosity distributions in the sample are uncorrelated, we can marginalize over one variable to evaluate the marginalized QLF along the other direction, retaining a larger sample for the analysis. This is especially useful for small samples, such as ours. The assumption that the luminosity (absolute magnitude) and redshift distributions of the sample are uncorrelated is 
Table 4

The Binned QLF

\begin{tabular}{lccccc}
\hline \hline $\begin{array}{l}M_{1450} \\
(\mathrm{mag})\end{array}$ & $N$ & $N_{\text {corr }}$ & $\begin{array}{c}\log _{10} \Phi \\
\left(\mathrm{Mpc}^{-3} \mathrm{mag}^{-1}\right)\end{array}$ & $\begin{array}{c}\sigma \Phi \\
\left(\mathrm{Gpc}^{-3} \mathrm{mag}^{-1}\right)\end{array}$ & $\begin{array}{c}\text { Bin } \\
\text { Filled }\end{array}$ \\
\hline $2.8 \leqslant z<3.0$ & & & & \\
\hline-28.9 & 2 & 3.9 & -9.46 & 0.25 & True \\
-28.5 & 4 & 10.1 & -9.05 & 0.50 & True \\
-28.15 & 11 & 28.6 & -8.47 & 1.08 & True \\
-27.85 & 7 & 21.2 & -8.60 & 1.00 & True \\
-27.6 & 9 & 41.3 & -8.13 & 2.59 & True \\
\hline $3.0 \leqslant z<3.5$ & & & & \\
\hline-28.9 & 3 & 3.9 & -9.85 & 0.08 & True \\
-28.5 & 10 & 12.9 & -9.33 & 0.15 & True \\
-28.15 & 28 & 42.0 & -8.69 & 0.39 & True \\
-27.85 & 31 & 67.6 & -8.48 & 0.60 & True \\
-27.6 & 17 & 69.9 & -8.29 & 1.28 & False \\
\hline $3.5 \leqslant z<4.0$ & & & & \\
\hline-28.9 & 2 & 2.1 & -10.09 & 0.06 & True \\
-28.5 & 6 & 7.2 & -9.56 & 0.11 & True \\
-28.15 & 12 & 18.8 & -9.02 & 0.28 & True \\
-27.85 & 6 & 15.4 & -9.08 & 0.34 & False \\
-27.6 & 2 & 9.3 & -8.68 & 1.49 & False \\
\hline $4.0 \leqslant z<4.5$ & & & & \\
\hline-28.9 & 2 & 2.3 & -10.04 & 0.06 & True \\
-28.5 & 5 & 7.8 & -9.50 & 0.14 & True \\
\hline & & & & &
\end{tabular}

Table 5

Fit Parameters of the Maximum Likelihood Estimation for the QLF

\begin{tabular}{lcrrr}
\hline \hline$z$ & $\begin{array}{c}M_{1450}^{*} \\
\text { (mag) }\end{array}$ & $\begin{array}{c}\log _{10}\left[\Psi_{0}^{\star}\right] \\
\left(\mathrm{Mpc}^{-3} \mathrm{mag}^{-1}\right)\end{array}$ & $\gamma$ & $\beta$ \\
\hline $2.8-4.5$ & -31.5 to -27 & $-4.88_{-0.32}^{+0.32}$ & $-0.38_{-0.11}^{+0.10}$ & $-4.08_{-0.19(0.59)}^{+0.19(0.54)}$ \\
\hline $3.0-4.5$ & -31.5 to -27 & $-4.59_{-0.41}^{+0.42}$ & $-0.43_{-0.13}^{+0.13}$ & $-4.17_{-0.22(0.68)}^{+0.21(0.62)}$ \\
\hline $2.8-4.5$ & -31.5 to -28 & $-4.58_{-0.56}^{+0.57}$ & $-0.36_{-0.15}^{+0.15}$ & $-4.44_{-0.38(1.23)}^{+0.36(1.01)}$ \\
\hline $3.0-4.5$ & -31.5 to -28 & $-4.44_{-0.47}^{+0.48}$ & $-0.40_{-0.13}^{+0.12}$ & $-4.46_{-0.35(1.12)}^{+0.33(0.94)}$
\end{tabular}

identical to assuming an underlying QLF of the form

$$
\Psi\left(M_{1450}, z\right)=\rho(z) \times \psi\left(M_{1450}\right) .
$$

We have introduced the methodology in the previous ELQS paper and refer all interested readers to Sections 6.2 and 6.3 of Paper II.

To test whether the redshifts and luminosities of the full ELQS sample can be regarded as uncorrelated, we perform a standard correlation test (Efron \& Petrosian 1992; Maloney \& Petrosian 1999; Fan et al. 2001) and calculate the $\tau$ statistic (Paper II, Section 6.2). As long as $|\tau| \lesssim 1$, both variables can be regarded as uncorrelated parameters at the $\sim 1 \sigma$ level and can be treated independently.

For the full ELQS QLF sample we obtain $\tau=-0.34$ $\left(\tau=-0.19 ; M_{1450} \leqslant-27.7\right)$ and can therefore proceed with the calculation of the differential marginal distributions. If we restrict the sample to higher redshifts $(3.0 \leqslant z \leqslant 4.5)$, $\tau$ increases to $-1.09\left(\tau=-1.24 ; M_{1450} \leqslant-27.7\right)$.

The differential marginal distributions can be calculated using Lynden-Bell's $C^{-}$estimator (Lynden-Bell 1971). We have modified the $C^{-}$estimator algorithm offered by the astroML ${ }^{11}$ library (see also Ivezić et al. 2014) to incorporate arbitrary selection functions (Fan et al. 2001). We compute the normalized differential distributions in absolute magnitude $\psi\left(M_{1450}\right)$ and redshift $\rho(z)$ with errors estimated on 20 bootstrap samples of our data.

The marginal differential magnitude distribution $\psi\left(M_{1450}\right)$, the number density of quasars as a function of magnitude, is calculated in the same magnitude bins we have chosen for the binned luminosity function in Section 6.1 (starting with $\left.M_{1450}=-27.7\right)$. It is normalized by

$$
\Phi\left(M_{1450} \leqslant-27.7\right) \equiv \int_{-\infty}^{-27.7} \psi\left(M_{1450}\right) d M_{1450},
$$

the total number of quasars with $M_{1450} \leqslant-27.7$. We estimate the slope of the resulting distribution by fitting a single power law, $\log _{10}\left(\psi\left(M_{1450}\right)\right) \propto-0.4(\beta+1) M_{1450}$, to the data. Over the entire redshift range, $2.8 \leqslant z \leqslant 4.5$, we find the slope to be best fit by $\beta=-4.45 \pm 0.23$.

The marginal differential redshift distribution $\rho(z)$, the spatial density of quasars as a function of redshift, uses the same redshift bins as our binned QLF analysis in Section 6.1. To analyze the evolution of the spatial density with redshift we use an exponential model, $\log _{10}(\rho(z)) \propto \gamma z$, and fit it to the data. We find a value of $\gamma=-0.41 \pm 0.02$ over the entire redshift range of our sample. The marginal differential distributions as well as their parametric fits are displayed in Figure 5.

It should be noted that the parametric fits to both distributions combined resemble a single power-law model for the QLF with exponential density evolution. We use this model in the following section to perform maximum likelihood fits.

\subsection{Maximum Likelihood Estimation of the QLF}

In this section we will calculate parametric maximum likelihood fits to the ELQS QLF sample without constraining it to redshift or magnitude bins. This analysis revisits Section 6.4 of Paper II with the full ELQS sample.

We follow Marshall et al. (1983) in calculating the maximum likelihood for the QLF $\Psi(M, z)$ by minimizing the $\log$ likelihood function

$$
\begin{aligned}
S= & -2 \sum_{i}^{N} \ln \left(\Psi\left(M_{i}, z_{i}\right)\left(p\left(M_{i}, z_{i}\right)\right)\right. \\
& +2 \iint \Psi(M, z) p(M, z) \frac{d V}{d z} d M d z .
\end{aligned}
$$

Confidence intervals on all parameters are derived from the likelihood function $S$ by using a $\chi^{2}$ distribution in $\Delta S=S-S_{\min }$ (Lampton et al. 1976).

In most cases the QLF can be well represented by a DPL (Boyle et al. 1988) at $z \lesssim 4$,

$$
\Psi(M, z)=\frac{\Psi^{\star}}{10^{0.4(\alpha+1)\left(M-M^{\star}\right)}+10^{0.4(\beta+1)\left(M-M^{\star}\right)}} .
$$

The four parameters, $\Psi^{\star}$ the overall normalization, $M^{\star}$ the break magnitude between the power laws, $\alpha$ and $\beta$ the faintand bright-end slopes, define the QLF and are known to evolve with redshift.

\footnotetext{
${ }^{11}$ https://github.com/astroML
} 

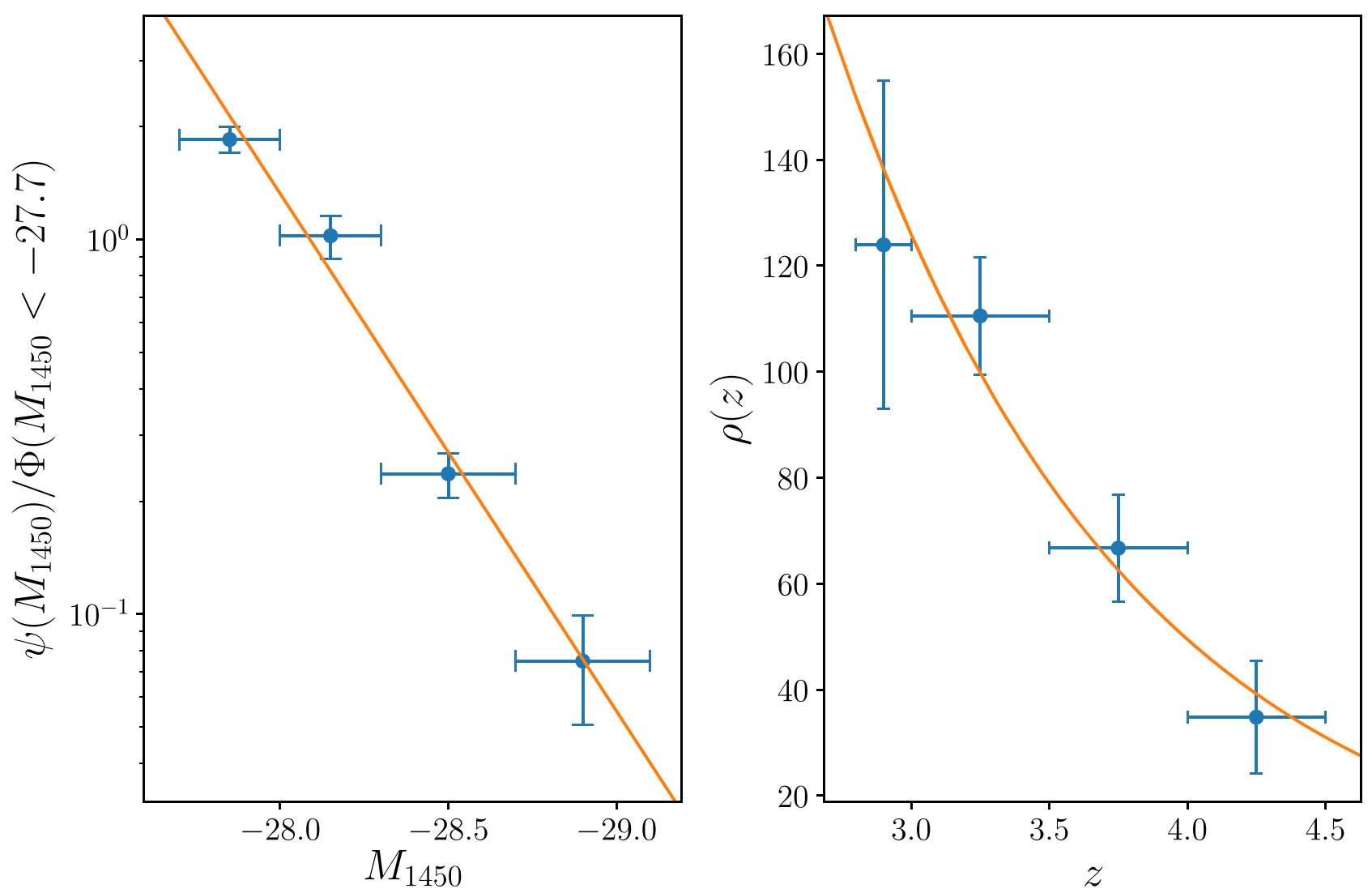

Figure 5. Left: the normalized marginal differential distribution of the QLF $\psi\left(M_{1450}\right) / \Phi\left(M_{1450} \leqslant-27.7\right)$ as a function of absolute magnitude $M_{1450}$. The error bars in the magnitude direction represent the bin width, while the errors in $\psi\left(M_{1450}\right)$ show the $1 \sigma$ statistical error margins from the bootstrap sampling. The orange line is the maximum likelihood fit to the data points, $\psi\left(M_{1450}\right) \propto 10^{-0.4(-4.45+1) M_{1450}}$. Right: the spatial density of the QLF $\rho(z)$ as a function of redshift $z$. The error bars in the redshift direction show the width of the redshift bins, while the error bars along $\rho(z)$ show the $1 \sigma$ statistical error margins from the bootstrap sampling. The orange line is the maximum likelihood fit to the data points, $\log _{10}(\rho(z)) \propto-0.41 z$.

The break magnitude, for example, has been shown to evolve strongly from $M_{1450}^{\star} \approx-25.6$ at $z=2.8$ to $M_{1450}^{\star} \approx-26.5$ at $z=4.5$ (see McGreer et al. 2013, their Figure 19). Therefore the ELQS sample, which probes only the luminous end of the quasar population $\left(M_{1450}^{\star} \lesssim-27\right)$, does not constrain the break magnitude $M^{\star}$ nor the faint-end slope $\alpha$. For this reason we assume a fixed break magnitude of $M_{1450}^{\star}=-26$ and parameterize the QLF using only a single power law,

$$
\Psi(M, z)=\Psi^{\star}(z) \times 10^{-0.4(\beta+1)\left(M-M_{1450}^{\star}\right)} .
$$

We include redshift evolution by allowing the normalization $\Psi^{\star}(z)$ to vary as an exponential function of redshift,

$$
\log _{10}\left[\Psi^{\star}(z)\right]=\log _{10}\left[\Psi_{0}^{\star}\right]+\gamma z .
$$

Here $\Psi_{0}^{\star}$ is the normalization at $z=0$ and $\gamma$ is a parameter of the exponential redshift evolution.

The independent redshift and magnitude evolution is supported over the full redshift range $z=2.8-4.5$ as shown by our analysis in Section 6.2.

The maximum likelihood fits are calculated using the simqso package (McGreer et al. 2013). We remind the reader that our ELQS QLF sample is reduced from 407 to 166 quasars by the photometric criteria we have used for the calculation of our selection function.

The parametric fits are calculated for the entire sample as well as for three subsamples constrained in redshift and/or absolute magnitude $M_{1450}$ as listed in the first two columns of Table 5. These ranges also serve as the integration boundaries for the calculation of $S$ in Equation (5). The remaining columns of Table 5 list the best-fit values for the three fit parameters including their $1 \sigma$ statistical uncertainties. In the case of $\beta$ we have also included the $3 \sigma$ uncertainties in parenthesis. The maximum likelihood fit over the entire range of redshift and magnitude (first row of Table 5) is also shown as the red solid line in Figure 4.

For the entire sample (first row in Table 5), we find the brightend slope to be steep with $\beta=-4.08$. This value is somewhat steeper than our estimate from the ELQS-N sample (Table 5 of Paper II), $\beta=-3.96$, but lies well within the $1 \sigma$ uncertainties. The single power-law fits constrain the bright-end slope at $z=2.8-4.5$ to $\beta \leqslant-3.4$ with $99 \%$ confidence. The best-fit results for the exponential density evolution, $\log _{10}\left[\Psi_{0}^{\star}\right]=-4.88$ and $\gamma=-0.38$, describe a moderately decreasing density similar to our previous estimate for the ELQS-N sample.

If we limit the ELQS QLF sample to higher redshifts (second and fourth rows in Table 5), the bright-end slope and the density evolution steepen slightly. Imposing a faint limit of $M_{1450}=-28$ leads to a steepening of the bright-end slope, while the density evolution becomes slightly more moderate. The dependence of the bright-end slope on the sampled magnitude range potentially indicates that the break magnitude is brighter than anticipated, and is therefore influencing our QLF estimate. Alternatively, this effect could signal a deviation from a simple power law at the bright end. 
Table 6

Results of Maximum Likelihood Double Power-law Fits to the ELQS QLF Sample Assuming a Fixed Faint-end Slope $(\alpha)$ and Break Magnitude $\left(M_{1450}^{*}\right)$

\begin{tabular}{|c|c|c|c|c|}
\hline $\begin{array}{l}M_{1450}^{*} \\
(\mathrm{mag})\end{array}$ & $\alpha$ & $\beta$ & $\begin{array}{c}\log _{10}\left[\Psi_{0}^{\star}\right] \\
\left(\mathrm{Mpc}^{-3} \mathrm{mag}^{-1}\right)\end{array}$ & $\gamma$ \\
\hline-26.0 & -2.00 & $-4.12_{-0.18}^{+0.18}$ & $-4.88_{-0.31}^{+0.32}$ & $-0.36_{-0.10}^{+0.10}$ \\
\hline-26.0 & -1.90 & $-4.16_{-0.18}^{+0.18}$ & $-5.41_{-0.32}^{+0.32}$ & $-0.38_{-0.11}^{+0.10}$ \\
\hline-26.0 & -1.80 & $-4.15_{-0.18}^{+0.18}$ & $-5.42_{-0.32}^{+0.32}$ & $-0.38_{-0.11}^{+0.10}$ \\
\hline-26.0 & -1.70 & $-4.15_{-0.18}^{+0.18}$ & $-5.42_{-0.32}^{+0.32}$ & $-0.38_{-0.11}^{+0.10}$ \\
\hline-26.5 & -2.00 & $-4.17_{-0.18}^{+0.18}$ & $-5.40_{-0.32}^{+0.32}$ & $-0.38_{-0.11}^{+0.10}$ \\
\hline-26.5 & -1.90 & $-4.16_{-0.18}^{+0.18}$ & $-5.41_{-0.32}^{+0.32}$ & $-0.38_{-0.11}^{+0.10}$ \\
\hline-26.5 & -1.80 & $-4.15_{-0.18}^{+0.18}$ & $-5.42_{-0.32}^{+0.32}$ & $-0.38_{-0.11}^{+0.10}$ \\
\hline-26.5 & -1.70 & $-4.15_{-0.18}^{+0.18}$ & $-5.42_{-0.32}^{+0.32}$ & $-0.38_{-0.11}^{+0.10}$ \\
\hline-27.0 & -2.00 & $-4.30_{-0.18}^{+0.18}$ & $-5.95_{-0.32}^{+0.33}$ & $-0.38_{-0.11}^{+0.10}$ \\
\hline-27.0 & -1.90 & $-4.29_{-0.19}^{+0.18}$ & $-5.96_{-0.32}^{+0.33}$ & $-0.38_{-0.11}^{+0.10}$ \\
\hline-27.0 & -1.80 & $-4.28_{-0.18}^{+0.18}$ & $-5.96_{-0.32}^{+0.33}$ & $-0.38_{-0.11}^{+0.10}$ \\
\hline-27.0 & -1.70 & $-4.28_{-0.18}^{+0.18}$ & $-5.97_{-0.32}^{+0.33}$ & $-0.38_{-0.11}^{+0.10}$ \\
\hline-27.5 & -2.00 & $-4.60_{-0.21}^{+0.21}$ & $-6.46_{-0.33}^{+0.33}$ & $-0.38_{-0.11}^{+0.10}$ \\
\hline-27.5 & -1.90 & $-4.60_{-0.21}^{+0.21}$ & $-6.47_{-0.33}^{+0.33}$ & $-0.38_{-0.11}^{+0.10}$ \\
\hline-27.5 & -1.80 & $-4.60_{-0.21}^{+0.21}$ & $-6.47_{-0.33}^{+0.33}$ & $-0.38_{-0.11}^{+0.10}$ \\
\hline-27.5 & -1.70 & $-4.61_{-0.21}^{+0.21}$ & $-6.47_{-0.33}^{+0.33}$ & $-0.38_{-0.11}^{+0.10}$ \\
\hline-28.0 & -2.00 & $-5.24_{-0.31}^{+0.30}$ & $-6.88_{-0.33}^{+0.34}$ & $-0.40_{-0.10}^{+0.10}$ \\
\hline-28.0 & -1.90 & $-5.26_{-0.31}^{+0.30}$ & $-6.87_{-0.33}^{+0.34}$ & $-0.41_{-0.10}^{+0.10}$ \\
\hline-28.0 & -1.80 & $-5.29_{-0.31}^{+0.30}$ & $-6.86_{-0.33}^{+0.34}$ & $-0.41_{-0.10}^{+0.10}$ \\
\hline-28.0 & -1.70 & $-5.32_{-0.31}^{+0.30}$ & $-6.85_{-0.33}^{+0.34}$ & $-0.41_{-0.10}^{+0.10}$ \\
\hline
\end{tabular}

\subsection{DPL Fits}

To ascertain the influence of the break magnitude on our single power-law fits, we investigate how our data would be represented assuming a broken DPL for the QLF (Boyle et al. 1988; Pei 1995).

At first we perform QLF fits following the broken DPL (Equation (6)) with density evolution (Equation (8)), assuming a large range of fixed values for the break magnitude, $M_{1450}^{*}=-26.5,-27,-27.5,-28$, and the faint-end slope, $\alpha=-1.7,-1.8,-1.9,-2.0$. The choices for the fixed parameters are guided by previous works at lower and higher redshifts (Croom et al. 2009; McGreer et al. 2013; Ross et al. 2013; Yang et al. 2016). The best-fit parameters of the resulting 16 fits are listed in Table 6.

While the assumed faint-end slope does not have any strong effect on the three fitted parameters $\left(\beta, \log _{10}\left[\Psi_{0}^{\star}\right], \gamma\right)$, the break magnitude clearly does affect the bright-end slope and the normalization. For brighter assumed break magnitudes we obtain a lower normalization and a steeper bright-end slope, revealing the potential bias that our single power-law fits carry. The redshift evolution of the normalization, $\gamma$, is not affected by different assumptions for the break magnitude. The dependence of the bright-end slope and the normalization on the break magnitude is already well documented in the literature (McGreer et al. 2013; Yang et al. 2016) and is a degeneracy that arises from the functional form of the broken DPL. That our fit results are not affected by the choice of the faint-end slope only reflects that our data do not constrain the faint-end slope. Other studies of the QLF that constrain the faint-end slope find a dependence on the break magnitude (McGreer et al. 2013; Onoue et al. 2017).

In addition to the 16 DPL fits with fixed break magnitude and faint-end slope, we calculate a fit to a QLF model with additional evolution in the break magnitude (luminosity evolution),

$$
M_{1450}^{*}(z)=M_{1450}^{*}(z=2.9)+c(z-2.9) .
$$

This redshift parameterization of the QLF with separate luminosity evolution and density evolution is often abbreviated as LEDE. In our case we assume $M_{1450}^{*}(z=2.9)=-27.0$, $c=-0.3$, and $\alpha=-1.9$. The choice of $c$ was motivated by the binned QLFs of Richards et al. (2006) and Ross et al. (2013) at the faint end. We retrieve best-fit values of $\beta=-4.33_{-0.19}^{+0.18} \log _{10}\left[\Psi_{0}^{\star}\right]=-4.86_{-0.32}^{+0.32}$, and $\gamma=-0.75_{-0.10}^{+0.10}$. The value for the bright-end slope and the normalization are still consistent with our single power-law results, while the density evolution parameter $\gamma$ has steepened significantly. However, with the assumption of a relatively flat slope for the luminosity evolution, our results for the density evolution are similar to those of Yang et al. (2016), who fit a LEDE model at $z \sim 5$ to find $c=-0.5 \pm 0.08$ ( $c_{2}$ in their notation) and $\gamma=-0.81 \pm 0.03\left(c_{1}\right.$ in their notation).

Additionally, we perform broken DPL fits to the binned QLF of our sample complemented by the QLFs of Richards et al. (2006) at $z=3.5-4.5$ and Ross et al. (2013) at $z=2.8-3.5$. We show the best-fit results in Figure 6 and present the best-fit values in Table 7. The fit in the lowest redshift range, $z=2.8-3.0$, is dominated by the QLF data of Ross et al. (2013) as we contribute only one data point at the bright end. At the higher redshifts the binned ELQS QLF contributes substantially to the determination of the bright-end slope and the break magnitude because it extends the dynamic range in $M_{1450}$ by more than one magnitude toward the bright end.

At $z=3.0-4.5$ the best-fit break magnitudes have values of $M_{1450}^{*} \leqslant-27$ and are therefore not only in the magnitude range sampled by the ELQS, but also around one magnitude brighter than expected from the literature (McGreer et al. 2013). It is not surprising that the bright-end slopes of the broken DPL fits are subsequently steeper than the single power-law fits. At $z=3.0-3.5, z=3.5-4.0$, and $z=4.0-4.5$, we retain brightend slopes of $\beta=-4.58,-4.52$, and -4.50 for the broken DPL, which are significantly steeper than our best-fit single power-law slope of $\beta \approx-4.1$.

These results strongly indicate that the ELQS extends to the break magnitude at $z=2.8-4.5$. Complementing the ELQS data with previous measurements of the binned QLF leads to the conclusion that the bright-end slope is even steeper than our single power-law fits suggested.

\section{Discussion}

Our analysis of the ELQS sample indicates a significantly steeper bright-end slope $(\alpha \approx-4.1$ to -4.7$)$ than previous studies at these redshifts suggested $(\alpha \approx-2.5$; Fan et al. 2001; Richards et al. 2006; Masters et al. 2012; Akiyama et al. 2018). In this section we will place our results in the context of other studies of the QLF across the whole redshift range probed.

In most cases a broken DPL form is fit to the QLF of UVbright type-I quasars in narrow redshift slices to determine the four fit parameters $\left(M_{1450}^{*}, \alpha, \beta, \log _{10}\left[\Psi^{\star}\right]\right)$ as a function of redshift. While the ELQS does not probe the faint end of the QLF, we have combined our binned ELQS QLF with previous measurements from the SDSS (Richards et al. 2006; Ross et al. 2013) to calculate all four fit parameters.

We exclude the redshift range $z=2.8-3.0$ from the following comparison, because the ELQS only contributes one data point 


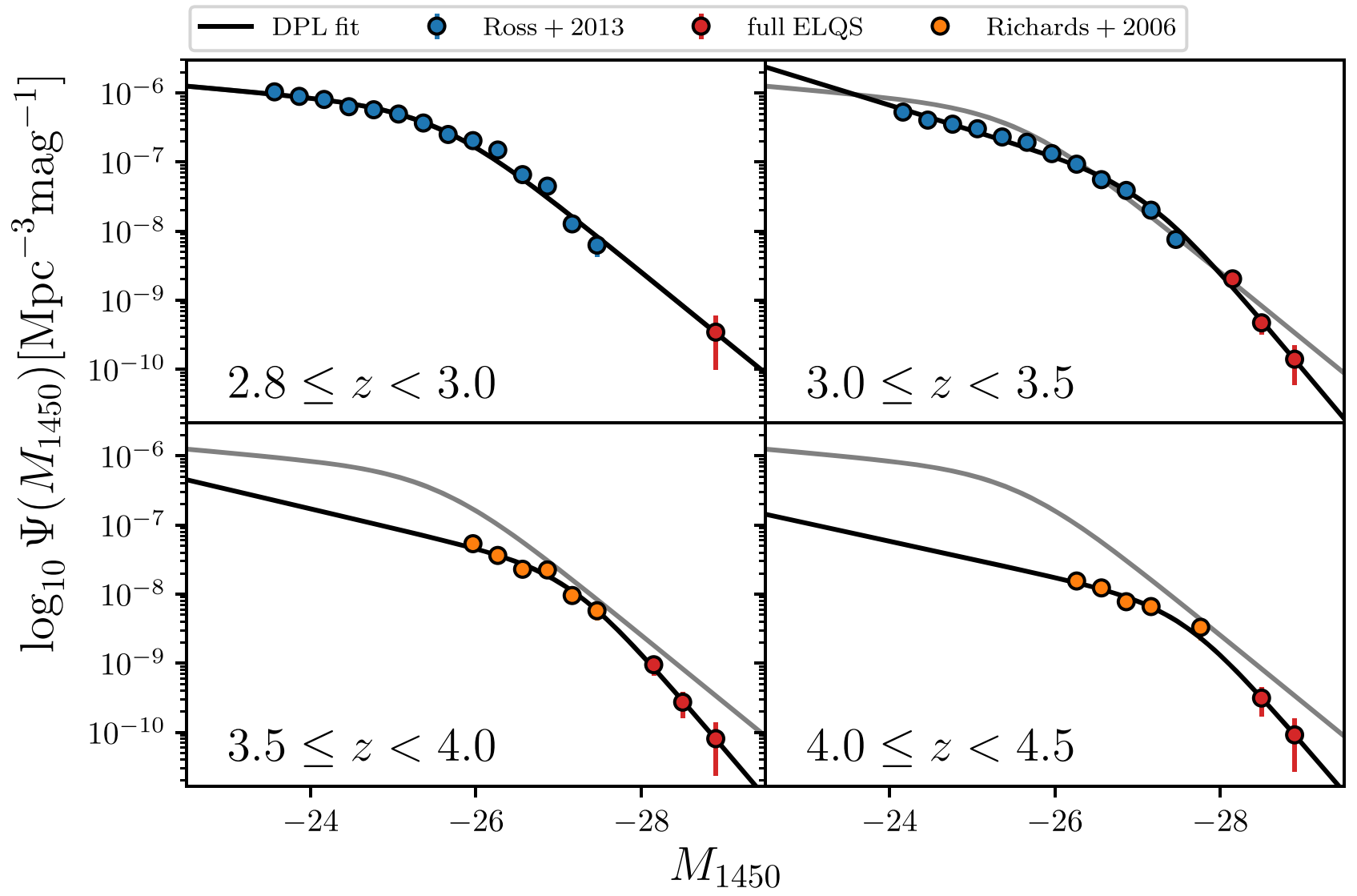

Figure 6. Broken DPL fits using a $\chi^{2}$-minimization to the binned QLFs from the ELQS (red) and the SDSS DR9 (Ross et al. 2013, blue) as well as the ELQS and the SDSS DR3 (Richards et al. 2006, orange) in the four redshift ranges sampled by the ELQS. In the lowest redshift range $(z=2.8-3.0)$ the single ELQS data point does not contribute significantly to the fit. However, in the three higher redshift ranges $(z=3.0-4.5)$ the ELQS data strongly help to constrain the bright-end slope. The best-fit values are given in Table 7. We further display the $z=2.8-3.0$ DPL fit as a gray line in the three higher redshift ranges for visual comparison.

Table 7

Result of DPL Fits ( $\chi^{2}$-minimization) to the Binned QLFs from ELQS/SDSS DR9 (Ross et al. 2013) and ELQS/SDSS DR3 (Richards et al. 2006) (see Figure 6)

\begin{tabular}{lccccrr}
\hline \hline$z$ & $\begin{array}{c}M_{1450}^{*} \\
(\mathrm{mag})\end{array}$ & $\alpha$ & $\beta$ & $\begin{array}{c}\log _{10}\left[\Psi_{0}^{\star}\right] \\
\left(\mathrm{Mpc}^{-3} \mathrm{mag}^{-1}\right)\end{array}$ & $\begin{array}{c}\sigma\left[\Psi_{0}^{\star}\right] \\
\left(\mathrm{Gpc}^{-3} \mathrm{mag}^{-1}\right)\end{array}$ \\
\hline $2.8-3.0$ & $-25.58 \pm 0.22$ & $-1.27 \pm 0.20$ & $-3.44 \pm 0.07$ & -6.23 & 185.93 & ELQS Combined With \\
$3.0-3.5$ & $-27.13 \pm 0.21$ & $-1.92 \pm 0.16$ & $-4.58 \pm 0.18$ & -7.33 & 22.07 & Ross et al. (2013) \\
$3.5-4.0$ & $-27.17 \pm 0.28$ & $-1.70 \pm 0.66$ & $-4.52 \pm 0.15$ & -7.65 & 15.51 & Ross et al. (2013) \\
$4.0-4.5$ & $-27.57 \pm 0.24$ & $-1.65 \pm 0.46$ & $-4.50 \pm 0.18$ & -8.16 & 3.96 & Richards et al. (2006) \\
\hline
\end{tabular}

with reasonably large uncertainties to the fit. Therefore the best-fit results only reflect the data of Ross et al. (2013).

Figure 7 shows our best-fit results (red diamonds) compared to a variety of other studies. Open data points illustrate values that were held fixed in the fitting process. At the lowest redshifts we display the data of Stripe 82 in Ross et al. (2013) (purple dots). At intermediate redshifts we compare our data with the studies of Masters et al. (2012) (blue circles) and Akiyama et al. (2018) (blue triangles). The former study is focused on a faint sample of known quasars and photometric quasar candidates from the Cosmic Evolution Survey (COSMOS), which has to rely on the SDSS DR3 quasar sample of Richards et al. (2006) at the bright end. Similarly, Akiyama et al. (2018) probe the faint end of the $z \approx 4$ QLF using the Hyper Suprime-Cam Wide Survey and use data from the SDSS
DR7 to extend their sample to the bright end. At $z \sim 5$ we compare to data from Yang et al. (2016) and McGreer et al. (2018) (turquoise squares and diamonds, respectively). The former study constrains the faint-end slope, while the latter analyses the bright end. All light green data are from studies at $z \sim 6$ (Willott et al. 2010; Jiang et al. 2016; Onoue et al. 2017; Matsuoka et al. 2018). The data are slightly offset in redshift for display purposes. For some of the parameters at the highest redshifts uncertainties are not given in the corresponding publication. Kulkarni et al. (2018) have recently reanalyzed a multitude of quasar samples across $z=0-7.5$ to determine the evolution of the QLF in a homogeneous way. For their analysis of the QLF evolution they exclude data at the lowest redshifts $(z \leqslant 0.5)$ and around $z=2.4-3.8$, where they argue that the quasar samples are significantly affected by observational 

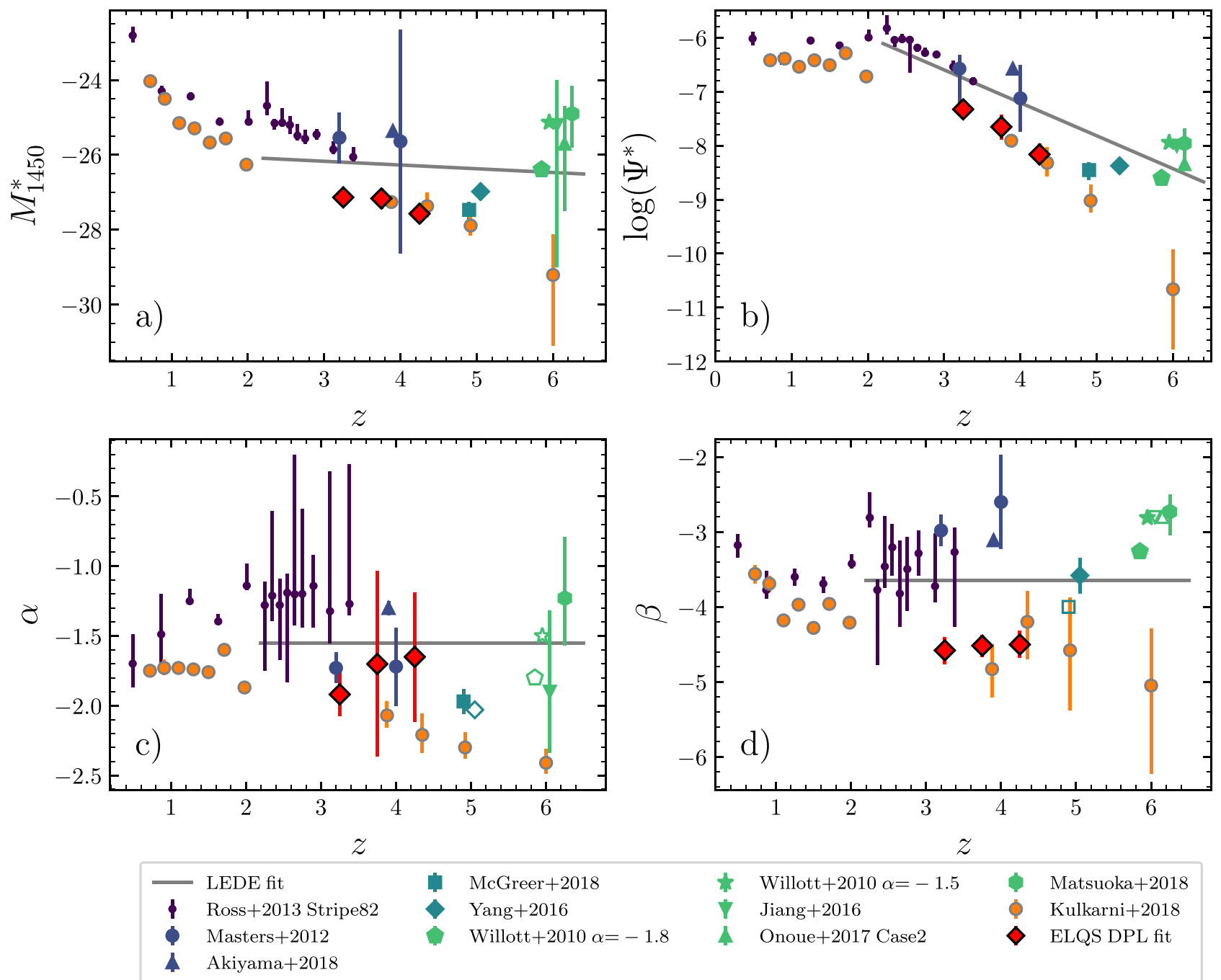

$\begin{array}{ll} & \text { McGreer }+2018 \\ & \text { Yang }+2016 \\ & \text { Willott }+2010 \alpha=-1.8\end{array}$

$\begin{array}{llll}\star & \text { Willott }+2010 \alpha=-1.5 & \diamond & \text { Matsuoka }+2018 \\ \text { Jiang }+2016 & \text { Onoue+2017 Case } 2 & \diamond & \text { ELQS DPL fit } \\ & & & \end{array}$

Figure 7. QLF parameters for fits of a broken DPL in narrow redshift bins. Solid data points are results from fits to data, while open data points symbolize fixed values in the QLF fit. We compare our DPL fit results (red diamonds, see Figure 6 and Table 7) with a variety of other studies across the whole redshift range (Willott et al. 2010; Masters et al. 2012; Ross et al. 2013; Jiang et al. 2016; Yang et al. 2016; Onoue et al. 2017; Akiyama et al. 2018; Kulkarni et al. 2018; Matsuoka et al. 2018; McGreer et al. 2018). In addition, the LEDE best-fit results (Section 7.1) are displayed as the gray solid line. (a) The break magnitude, $M_{1450}^{*}$, which brightens with increasing redshift. (b) The density normalization, $\log _{10}\left[\Psi^{\star}\right]$, which decreases strongly with increasing redshift. (c) The faint-end slope, $\beta$, which shows no systematic trend with redshift. (d) The bright-end slope, which also shows no consistent redshift-dependent behavior.

biases. We display their DPL fit parameters as orange circles at the redshifts unaffected by these systematics (see filled symbols in their Figure 4).

Figure 7(a) displays the redshift evolution of the break magnitude. Following the data of Ross et al. (2013), Yang et al. (2016), and McGreer et al. (2018), one can make out a clear trend of the break magnitude decreasing with increasing redshift. The data of Masters et al. (2012) and the studies at the highest redshift have significant error bars (where available), possibly allowing for the general trend to be continued up to $z \sim 6$. Our best-fit break magnitudes are clearly offset from the general trend by about one magnitude toward the brighter end. However, they are in good agreement with the data of Kulkarni et al. (2018).

The normalization, $\log _{10}\left(\Psi^{*}\right)$, shown in Figure $7(\mathrm{~b})$, decreases strongly with increasing redshift. The results of Kulkarni et al. (2018) generally follow the same trend as the other literature values until $z \sim 5$, while lying lower at all redshifts. They differ substantially from the results at $z \sim 6$, which is a consequence of the brighter break magnitude as we
Table 8

LEDE Model Fit Parameters

\begin{tabular}{lc}
\hline \hline Best-fit Parameter & Best-fit Value \\
\hline $\log _{10}\left[\Psi_{0}^{*}\right](z=2.2)$ & $-6.11 \pm 0.03$ \\
$M_{1450}^{*}(z=2.2)$ & $-26.09 \pm 0.05$ \\
$\alpha$ & $-1.55 \pm 0.02$ \\
$\beta$ & $-3.65 \pm 0.06$ \\
$c_{1}$ & $-0.61 \pm 0.02$ \\
$c_{2}$ & $-0.1 \pm-0.03$ \\
\hline
\end{tabular}

discuss later. Our best-fit results lie below the general trend and therefore agree well with the data of Kulkarni et al. (2018). The best-fit value of the normalization is strongly dependent on the break magnitude. The agreement of our data with the results of Kulkarni et al. (2018) is therefore not surprising.

Figure 7(c) shows the faint-end slope of the different studies as a function of redshift. The data do not suggest a strong evolution of the faint-end slope with redshift. While the purple 
data (Ross et al. 2013) suggest that the faint-end slope seems to be flattening with redshift, this trend is not supported by the other literature. The data of Kulkarni et al. (2018) even show the faint-end slope to be steepening with redshift. We retain faint-end slopes around $\alpha \approx-1.8$, which show considerable uncertainties. However, these values are in general agreement with the other data.

In Figure 7(d) we compare the best-fit bright-end slope of our work with the other values in the literature. Similarly to the faint-end slope there is no evident evolution of the bright-end slope with redshift. Some earlier studies reported a flattening of the bright-end slope from $z=0$ toward $z \approx 4$ (Fan et al. 2001; Richards et al. 2006). The data of Masters et al. (2012) and Akiyama et al. (2018) seem to support this claim, while our results and the data of Kulkarni et al. (2018) do not support it and rather argue for a consistently steep bright-end slope.

Figure 6 underlines the importance of the ELQS sample to the determination of the QLF compared to the original samples of Richards et al. (2006) and Ross et al. (2013). Because we extended the bright end by one magnitude, we are now able to securely constrain the bright-end slope and the break magnitude. The break magnitude is about one magnitude brighter than previously expected (McGreer et al. 2013; Yang et al. 2016), which has a strong impact on the measured faintand bright-end slopes. As a result we find best-fit bright-end slopes around $\beta \approx-4.6$ over $z=3.0-4.5$.

Our results generally agree well with the recent re-estimation of the QLF of Kulkarni et al. (2018). In their work the authors combine a large range of quasar samples to study the QLF evolution from $z=0$ up to $z=7.5$. In the redshift range probed by the ELQS they rely on the SDSS DR7 quasar sample (Schneider et al. 2010), the SDSS DR9 quasar sample (Ross et al. 2013), and the quasar sample of Glikman et al. (2011). The ELQS quasar sample overlaps strongly with the SDSS DR7 and DR9 quasar samples because we cover the same footprint. However, our novel quasar selection is independent of the SDSS quasar selection methodology. Therefore, the ELQS QLF analysis can be considered as an independent measurement with regard to the work of Kulkarni et al. (2018).

If we were to assume the evolution of the break magnitude evident in the study of Kulkarni et al. (2018) and our work, the break magnitude would reach values of $M_{1450}^{*} \approx-29$ at $z=6$, making constraints on the bright-end slope above $z \approx 6$ increasingly inaccessible. However, how does one reconcile this result and the studies at the highest redshifts (Matsuoka et al. 2018; Wang et al. 2018) that find the QLF well represented by a DPL with a much lower break magnitude around $M_{1450}^{*} \approx-25$ and a flatter bright-end slope around $\beta \approx-2.6$ ? Is their bright-end slope the faint-end slope at lower redshifts, and thus we need to introduce a broken triple power law for the QLF? Or is there an entirely different functional form that can describe the QLF better across all redshifts? While the recent results allowed us to raise these questions, an obvious solution is not yet in sight.

\subsection{Comparison to an Evolutionary QLF Model Fit}

We perform an evolutionary fit to the binned QLF at higher redshifts $(z>2.2)$ to compare with the DPL fits of the ELQS at $z=2.8-4.5$. For this analysis we supplement the binned QLF data used above for the DPL fits with the most recent data at lower (Palanque-Delabrouille et al. 2016) and higher (Akiyama et al. 2018; Matsuoka et al. 2018; McGreer et al. 2018) redshift.
To describe the evolution of the QLF across larger redshift ranges it is common practice to introduce redshift dependences on the parameters of the DPL form. We adopt an independent luminosity and density evolution model (LEDE, see also Ross et al. 2013) to describe the redshift dependence of the DPL. The LEDE model has been successful in describing the evolution of the QLF at higher redshifts (Ross et al. 2013; Palanque-Delabrouille et al. 2016).

We adopt the parameterization of Ross et al. (2013) and model the evolution of the normalization and break magnitude as

$$
\log _{10}\left[\Psi_{0}^{*}\right](z)=\log _{10}\left[\Psi_{0}^{*}\right](z=2.2)+c_{1}(z-2.2)
$$

and

$$
M_{1450}^{*}(z)=M_{1450}^{*}(z=2.2)+c_{2}(z-2.2) \text {. }
$$

The QLF is then fully described by the normalization at $z=2.2, \log _{10}\left[\Psi_{0}^{*}\right](z=2.2)$, the break magnitude at $z=2.2$, $M_{1450}^{*}(z=2.2)$, the power-law slopes $\alpha$ and $\beta$, and the two evolutionary parameters, $c_{1}$ and $c_{2}$.

We perform maximum likelihood fits to the binned QLF data using emcee (Foreman-Mackey et al. 2013) for Markov chain Monte Carlo sampling of the parameter space. We adopt the median values of the posterior distributions as our best-fit values and summarize them in Table 8.

We show the LEDE best-fit model (solid black line) in Figure 8 compared to the binned QLF data, our DPL fits in individual redshift ranges (solid red line), and the best-fit DPLs of the high-redshift QLFs (solid colored lines) according to their respective studies. While the LEDE fit seems to be an adequate representation of the binned QLF data, it deviates from the DPL fits in the specific redshift bins. If we compare the LEDE fit to the fit of McGreer et al. (2018) and our DPL fits (red solid lines, see Section 6.4), the LEDE model's break magnitude is fainter and its bright-end slope is less steep. This situation is reversed when comparing the LEDE model to Akiyama et al. (2018) and Matsuoka et al. (2018).

This comparison highlights the disparities between the different studies in different redshift ranges, which already became clear in Figure 7. In light of these differences in break magnitudes and bright- and faint-end slopes, it becomes increasingly challenging to find one model that coherently describes the redshift evolution of the QLF.

\section{Conclusions}

We have presented the motivation for the ELQS as well as our novel quasar selection using a $J-K-W 2$ color cut and machine learning methods (random forests) in Paper I. A subsequent publication, Paper II, reported our first spectroscopic observations in the North Galactic Cap (ELQS-N), constrained the ELQS selection function, and discussed a preliminary analysis of the QLF based on the ELQS-N quasar sample. With this work we conclude the ELQS. Spectroscopic follow-up of ELQS candidates has been mostly completed, allowing us to present the full ELQS quasar catalog (Section 5) and analyze the QLF on the full ELQS QLF sample.

1. We report the discovery of 70 new quasars (see Table 2 and Figure 9) at $z=2.8-4.5$ as part of the ELQS Southern Galactic Cap sample (ELQS-S). The full ELQS-S sample 


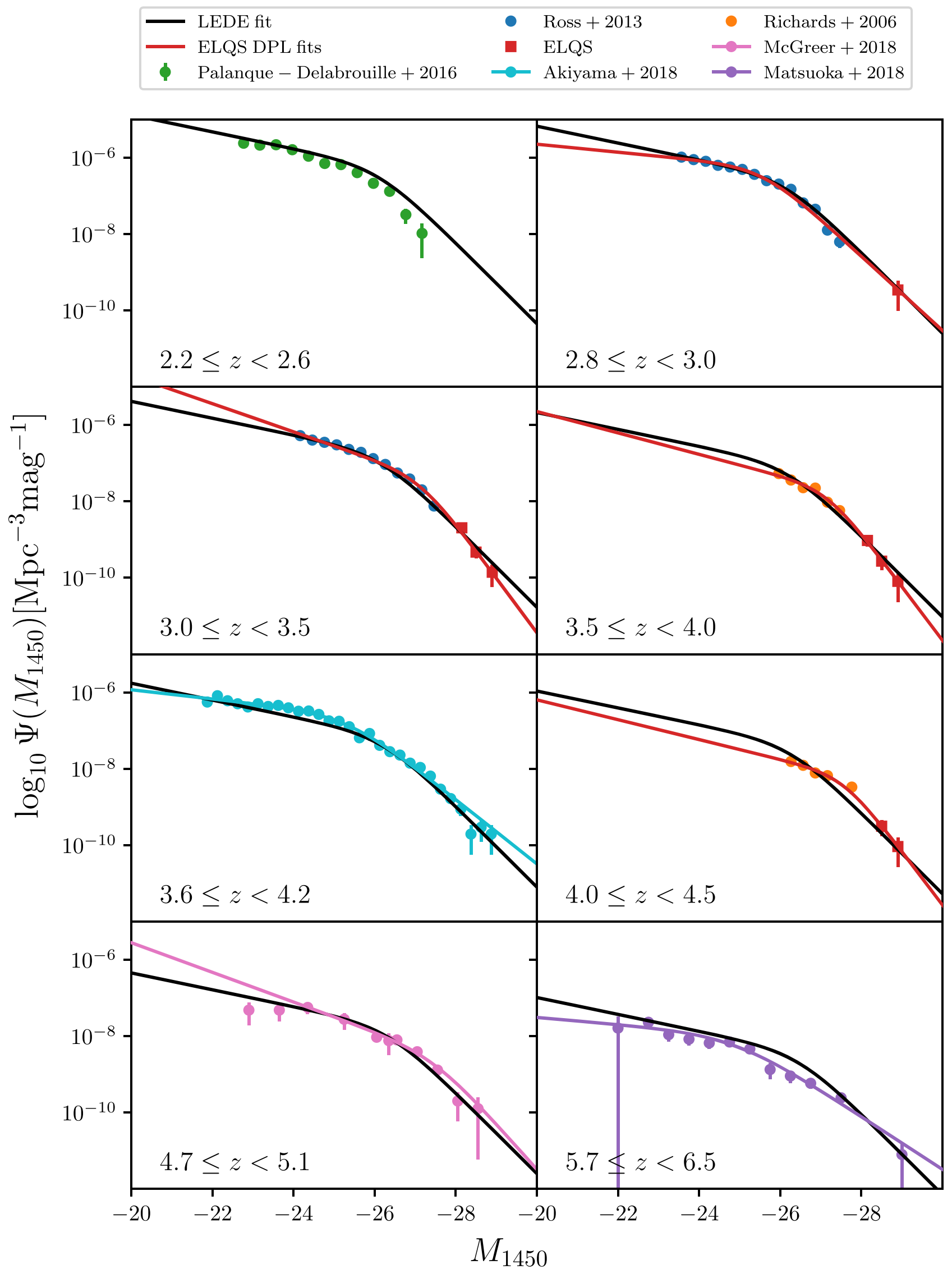

Figure 8. Results of the LEDE fit (solid black line) to binned QLF data (colored data points) across $z=2.2-6.5$. The binned QLF data are taken from a variety of recent studies (Richards et al. 2006; Ross et al. 2013; Palanque-Delabrouille et al. 2016; Akiyama et al. 2018; Matsuoka et al. 2018; McGreer et al. 2018). We further display the DPL fit to the binned QLF in individual redshift bins as solid lines, colored according to their reference. 
contains 137 quasars over an area of $4237.3 \pm 12.9 \mathrm{deg}^{2}$ of the SDSS footprint at R.A. $>270^{\circ}$ and R.A. $<90^{\circ}$. Our newly discovered quasars double the known population of quasars in the South Galactic Cap footprint of the SDSS survey. This sample improves upon the known SDSS spectroscopic incompleteness of the South Galactic Cap, allowing for a more unbiased measurement of quasars across the full SDSS footprint.

2. The full ELQS quasar catalog comprises 407 quasars of which 109 , or $\sim 26 \%$, are newly identified. Only 239 of the already known 298 quasars are part of SDSS DR14Q, which means that our quasar selection includes an additional 59 quasars from the literature missed by the SDSS quasar surveys. Overall our selection identified 509 primary quasar candidates, of which 407 were identified to be quasars, resulting in a selection efficiency of $\sim 80 \%$.

3. We have cross-matched the full ELQS sample to the AllWISE counterparts of the reprocessed ROSAT 2RXS catalog, GALEX GR6/7, and the FIRST survey. There are 11 sources with ROSAT 2RXS detections, which are all already known quasars in the literature. We identified 55 matches to GALEX sources, of which 14 are newly identified quasars with GALEX counterparts in either or both photometric UV bands. The rate of UV detections is about $13 \%$ for the full sample. We were also able to obtain $1.4 \mathrm{GHz}$ flux measurements from FIRST for 37 quasars in the full ELQS sample. Since the FIRST footprint has been chosen to overlap with the SDSS North Galactic Cap footprint, we evaluated the RLF for the ELQS-N sample. We use the $R$ parameter, the rest-frame ratio of the flux density at $6 \mathrm{~cm}(5 \mathrm{GHz})$ to flux density at $2500 \AA$, to classify quasars as radio-loud with $R \geqslant 10$. We estimate an $\operatorname{RLF}\left(m_{i} \leqslant 18\right) \approx(9.3 \pm 1.2) \%$ for the ELQS-N sample, which generally agrees with the value derived from the relation of Jiang et al. (2007) for our median redshift and absolute magnitude $\left(\mathrm{RLF}_{\mathrm{Jiang} 2007}=8.0 \%\right)$.

4. We further determine the fraction of quasars with BALs for the full ELQS sample. The DR12Q BAL flag (BAL_VI) provides information for 212 quasars, and we classify the remaining quasars by visual inspection or use previous classifications in the literature. A total of 23 quasars could not be identified due to the lack or the quality of the identification spectra. We could identify 79 BAL quasars out of 384 sources in the full ELQS sample. Of all newly identified quasars 17 display BAL features. We estimate an observed BAL quasar fraction of $\sim 21 \%$, which is large compared to other quasar samples (for example Trump et al. 2006; Maddox et al. 2008). It remains unclear whether the larger BAL quasar fraction is due to our observed-frame near-infrared-based selection, the sampled redshift range (redshift dependence, Allen et al. 2011) of the ELQS or our focus on the luminous end of the quasar distribution (luminosity dependence).

5. We evaluate the QLF based on the full ELQS sample in Section 6. A comparison of our binned QLF to the SDSS DR3 (Richards et al. 2006) and SDSS DR12 (Ross et al. 2013) QLF (see Figure 4) shows that the bright-end slope is steeper than the parametric fits to these two references suggest. We continue to analyze the differential marginal distributions of the QLF along the luminosity and redshift variables (Figure 5), finding a steep bright-end slope of $\beta \approx-4.45$ for a single power-law parameterization. A maximum likelihood fit to a single power-law QLF with exponential density evolution confirms these results with a best-fit bright-end slope of $\beta \approx-4.1$ for the full ELQS QLF sample. Our analysis further constrains the brightend slope to be steeper than $\beta \leqslant-3.4$ at the $3 \sigma$ level. Additionally, we perform broken DPL fits to the data to assess the possible bias introduced by a single power-law description. This analysis corroborates the steep values for the bright-end slopes. While earlier studies (Koo \& Kron 1988; Schmidt et al. 1995; Fan et al. 2001; Richards et al. 2006) suggested a flattening of the bright-end slope $\beta$ toward higher redshifts, all our analyses disfavor this scenario. In fact, our results in the intermediate redshift range rather indicate a consistent picture, in which the bright-end slope remains steep from the lowest redshifts (Croom et al. 2009; Ross et al. 2013) up to the highest (Jiang et al. 2008; Willott et al. 2010; McGreer et al. 2013; Yang et al. 2016) with some room for modest evolution.

6. We use a model of exponential density evolution to analyze the redshift evolution of the quasar number density. The differential marginal distribution and the maximum likelihood fit consistently indicate an exponential decline with $\gamma \approx-0.4$. Other studies at lower and higher redshifts (Fan et al. 2001; Ross et al. 2013; Yang et al. 2016) find a steeper decline of the quasar density toward higher redshift with $\gamma \sim-0.7$ to -0.5 . However, the uncertainties on our maximum likelihood fit would allow for $\gamma \approx-0.5$ at the $1 \sigma$ level.

7. We combine the binned ELQS QLF with values from the binned QLFs of Richards et al. (2006) and Ross et al. (2013) to calculate broken DPL fits over a larger magnitude range. Our best-fit results find the bright-end slope to be steep with values of $\beta \approx-4.6$ over $z=3.0-4.5$ and the break magnitude to be brighter by one magnitude compared to the previous literature. Only the recent reanalysis of a large combined sample of quasar surveys by Kulkarni et al. (2018) shows agreement with our results. We argue that the larger dynamic range in $M_{1450}$ probed by the ELQS survey at the bright end was crucial to constrain the bright-end slope properly because previous studies (Richards et al. 2006; Ross et al. 2013) did not sufficiently sample the population brighter than the break magnitude at these redshifts.

J.T.S., X.F., I.D.M., and J.Y. acknowledge support of US NSF grant AST-1515115 and NASA ADAP grant NNX17AF28G.

J.K.K. acknowledges financial support from the Danish Council for Independent Research (EU-FP7 under the MarieCurie grant agreement no. 600207) with reference DFFMOBILEX-5051-00115.

The Cosmic Dawn Center is funded by the DNRF.

Y.-L.W. is grateful for the support from the Heising-Simons Foundation. 
The work is in part based on observations made with the NOT, operated on the island of La Palma jointly by Denmark, Finland, Iceland, Norway, and Sweden, in the Spanish Observatorio del Roque de los Muchachos of the Instituto de Astrofisica de Canarias.

This publication makes use of data products from the Two Micron All Sky Survey, which is a joint project of the University of Massachusetts and the Infrared Processing and Analysis Center/California Institute of Technology, funded by the National Aeronautics and Space Administration and the National Science Foundation.

This publication makes use of data products from the Widefield Infrared Survey Explorer, which is a joint project of the University of California, Los Angeles, and the Jet Propulsion Laboratory/California Institute of Technology, funded by the National Aeronautics and Space Administration.

Funding for the Sloan Digital Sky Survey IV has been provided by the Alfred P. Sloan Foundation, the U.S. Department of Energy Office of Science, and the Participating Institutions. SDSS acknowledges support and resources from the Center for High-Performance Computing at the University of Utah. The SDSS website is www.sdss.org.

SDSS is managed by the Astrophysical Research Consortium for the Participating Institutions of the SDSS Collaboration including the Brazilian Participation Group, the Carnegie Institution for Science, Carnegie Mellon University, the Chilean Participation Group, the French Participation Group, HarvardSmithsonian Center for Astrophysics, Instituto de Astrofísica de Canarias, The Johns Hopkins University, Kavli Institute for the Physics and Mathematics of the Universe (IPMU)/University of Tokyo, Lawrence Berkeley National Laboratory, Leibniz Institut für Astrophysik Potsdam (AIP), Max-Planck-Institut für
Astronomie (MPIA Heidelberg), Max-Planck-Institut für Astrophysik (MPA Garching), Max-Planck-Institut für Extraterrestrische Physik (MPE), National Astronomical Observatories of China, New Mexico State University, New York University, University of Notre Dame, Observatório Nacional/MCTI, The Ohio State University, Pennsylvania State University, Shanghai Astronomical Observatory, United Kingdom Participation Group, Universidad Nacional Autónoma de México, University of Arizona, University of Colorado Boulder, University of Oxford, University of Portsmouth, University of Utah, University of Virginia, University of Washington, University of Wisconsin, Vanderbilt University, and Yale University.

This research has made use of the NASA/IPAC Extragalactic Database (NED), which is operated by the Jet Propulsion Laboratory, California Institute of Technology, under contract with the National Aeronautics and Space Administration.

This research made use of Astropy, a community-developed core Python package for Astronomy (Astropy Collaboration 2013, http://www.astropy.org). In addition, Python routines from scikit-learn (Pedregosa et al. 2011), SciPy (Jones et al. 2001), Matplotlib (Hunter 2007), lmfit (Newville et al. 2014; Oliphant 2007), and Pandas (McKinney 2010) were used in the quasar selection, data analysis, and creation of the figures.

\section{Appendix A The Full ELQS Quasar Catalog}

The ELQS quasar catalog is available as a machine-readable table (csv format) online. It has 55 columns, detailed in Table 9.

Table 9

Description of the Full ELQS Quasar Catalog Table

\begin{tabular}{|c|c|c|c|}
\hline Column & Column Name & Unit & Description \\
\hline 1 & WISE & $\cdots$ & Designation of the WISE AllWISE survey \\
\hline 2 & SDSS-RAdeg & $\operatorname{deg}$ & SDSS DR13 R.A., decimal degrees (J2000) \\
\hline 3 & SDSS-DEdeg & deg & SDSS DR13 decl., decimal degrees (J2000) \\
\hline 4 & RAh & $\mathrm{h}$ & Hour of SDSS DR13 R.A. (J2000) \\
\hline 5 & RAm & $\min$ & Minute of SDSS DR13 R.A. (J2000) \\
\hline 6 & RAs & $\mathrm{s}$ & Second of SDSS DR13 R.A. (J2000) \\
\hline 7 & DE- & $\cdots$ & Sign of SDSS DR13 decl. (J2000) \\
\hline 8 & DEd & deg & Degree of SDSS DR13 decl. (J2000) \\
\hline 9 & DEm & $\operatorname{arcmin}$ & Arcminute of SDSS DR13 decl. (J2000) \\
\hline 10 & DEs & $\operatorname{arcsec}$ & Arcsecond of SDSS DR13 decl. (J2000) \\
\hline 11 & WISE-RAdeg & deg & WISE R.A., decimal degrees (J2000) \\
\hline 12 & WISE-DEdeg & deg & WISE decl., decimal degrees (J2000) \\
\hline 13 & Ref & $\cdots$ & Reference to the quasar classification \\
\hline 14 & $\mathrm{z}-\mathrm{Ref}$ & $\cdots$ & Best redshift of the quasar according to the reference \\
\hline 15 & M1450 & mag & Absolute magnitude, $1450 \AA$, calculated using the K-correction determined in this work \\
\hline 16 & sel-prob & $\cdots$ & Selection probability according to our completeness calculation \\
\hline $17-26$ & [band]mag & mag & $\begin{array}{l}\text { Dereddened } A B \text { magnitudes of the SDSS DR13 ugriz, 2MASS } \mathrm{JHK}_{\mathrm{s}} \text { and AllWISE } W 1, W 2 \text { bands (bands }=[u, g, r, i, \\
z, J, H, K s, W 1, W 2]) \text {. It should be noted that all SDSS magnitudes are PSF magnitudes in the SDSS Asinh } \\
\text { magnitude system. }\end{array}$ \\
\hline $27-36$ & e_[band]mag & mag & $1 \sigma$ errors on the $A B$ magnitudes \\
\hline 37 & $E(B-V)$ & mag & $E(B-V)$ color excess \\
\hline 38 & $\mathrm{Ai}$ & mag & Extinction in the SDSS $i$-band \\
\hline 39 & FIRST & $\cdots$ & Boolean to indicate successful matches with the FIRST catalog \\
\hline 40 & Sep-FIRST & $\operatorname{arcsec}$ & Distance of the FIRST source relative to the SDSS position \\
\hline 41 & Flux-FIRST & $\mathrm{mJy} /$ beam & FIRST peak flux, mJy/beam \\
\hline 42 & e_Flux-FIRST & $\mathrm{mJy} /$ beam & rms error on the FIRST flux, $\mathrm{mJy} /$ beam \\
\hline 43 & GALEX & $\cdots$ & Boolean to indicate successful matches with the $G A L E X$ GR6/7 catalog \\
\hline 44 & Sep-GALEX & $\operatorname{arcsec}$ & Distance of the GALEX GR6/7 match relative to the SDSS position \\
\hline
\end{tabular}


Table 9

(Continued)

\begin{tabular}{|c|c|c|c|}
\hline Column & Column Name & Unit & Description \\
\hline 45 & NUVmag & mag & GALEX GR6/7 near-UV flux, magnitudes \\
\hline 46 & e_NUVmag & mag & Uncertainty in NUVmag \\
\hline 47 & FUVmag & mag & GALEX GR6/7 far-UV flux, magnitudes \\
\hline 48 & e_FUVmag & mag & Uncertainty in FUVmag \\
\hline 49 & TRXS & $\ldots$ & Boolean to indicate successful matches to the ROSAT 2RXS AllWISE counterparts \\
\hline 50 & Sep-TRXS & $\operatorname{arcsec}$ & $\begin{array}{l}\text { Match distance between the ELQS AllWISE position and the ROSAT 2RXS AllWISE position. The distance values are } \\
\text { often } 0 \text { or otherwise extremely small, because the positions match to numerical accuracy. }\end{array}$ \\
\hline 51 & f_TRXS & $\cdots$ & $\begin{array}{l}\text { A flag indicating the most probable AllWISE ROSAT 2RXS cross-match with } 1 \text {. This is the case for all matched } \\
\text { objects. }\end{array}$ \\
\hline 52 & Flux-TRXS & $\mathrm{erg} \mathrm{cm}^{-2} \mathrm{~s}^{-1}$ & 2RXS flux \\
\hline 53 & e_Flux-TRXS & $\mathrm{erg} \mathrm{cm}^{-2} \mathrm{~s}^{-1}$ & Uncertainty in Flux-TRXS \\
\hline 54 & BAL & $1 / 0 /-1$ & $\begin{array}{l}\text { Broad absorption line flag, indicating whether the object is visually identified as a BAL quasar }(1=\text { BAL quasars, } \\
0=\text { quasars, }-1=\text { no visual classification). }\end{array}$ \\
\hline 55 & QLF & $\ldots$ & Boolean to indicate whether the quasar is included in the estimation of the quasar luminosity function (Section 6). \\
\hline
\end{tabular}

(This table is available in its entirety in machine-readable form.)

\section{Appendix B \\ Discovery Spectra of the ELQS-S Quasars}

We present the discovery spectra for all newly discovered ELQS-S quasars in Figure 9. 


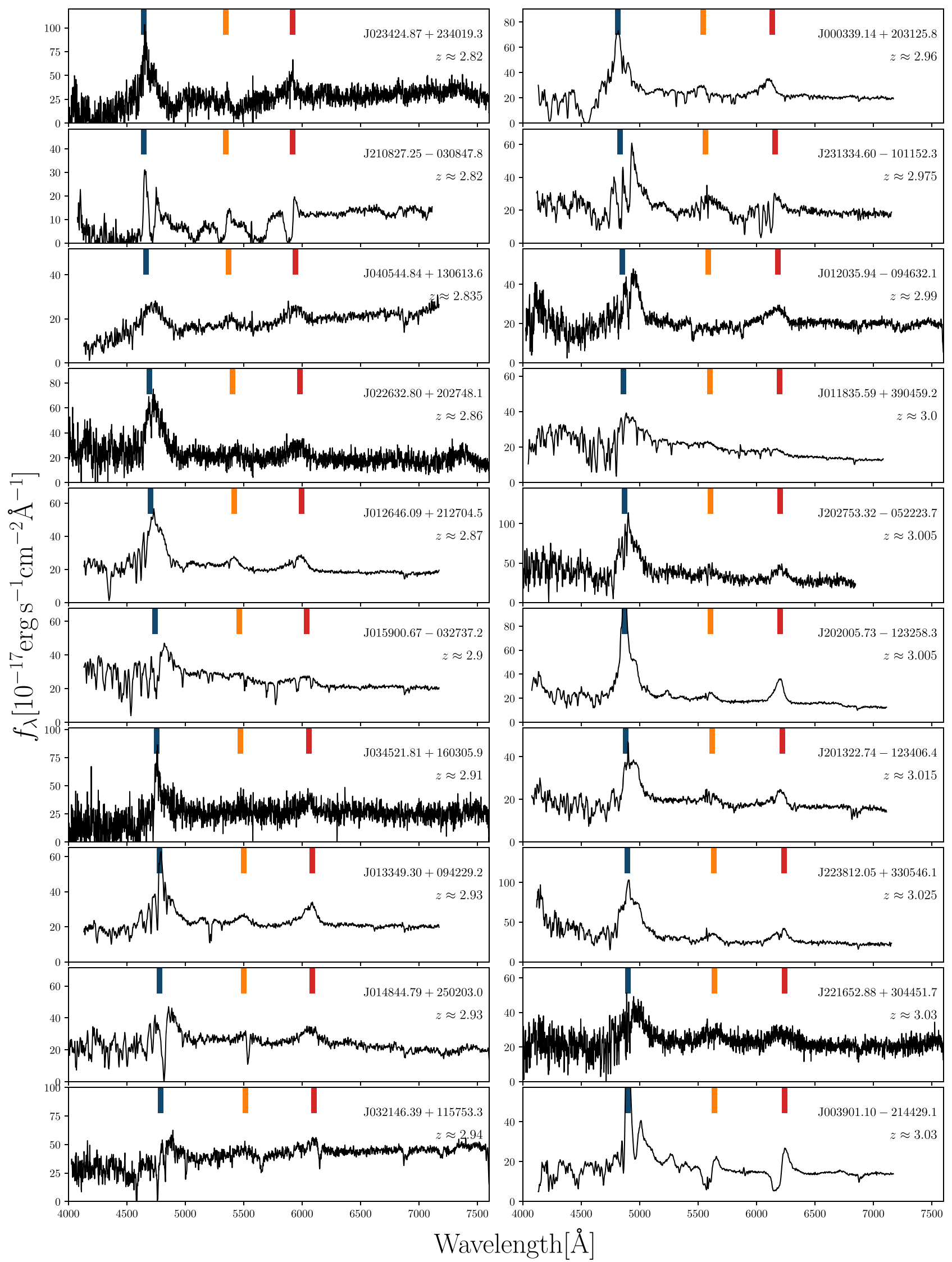

Figure 9. The discovery spectra of the newly identified ELQS-S quasars sorted by spectroscopic redshift. The dark blue, orange, and red bars denote the center positions of the broad Ly $\alpha$, Si IV, and C IV emission lines according to the spectroscopic redshift. 


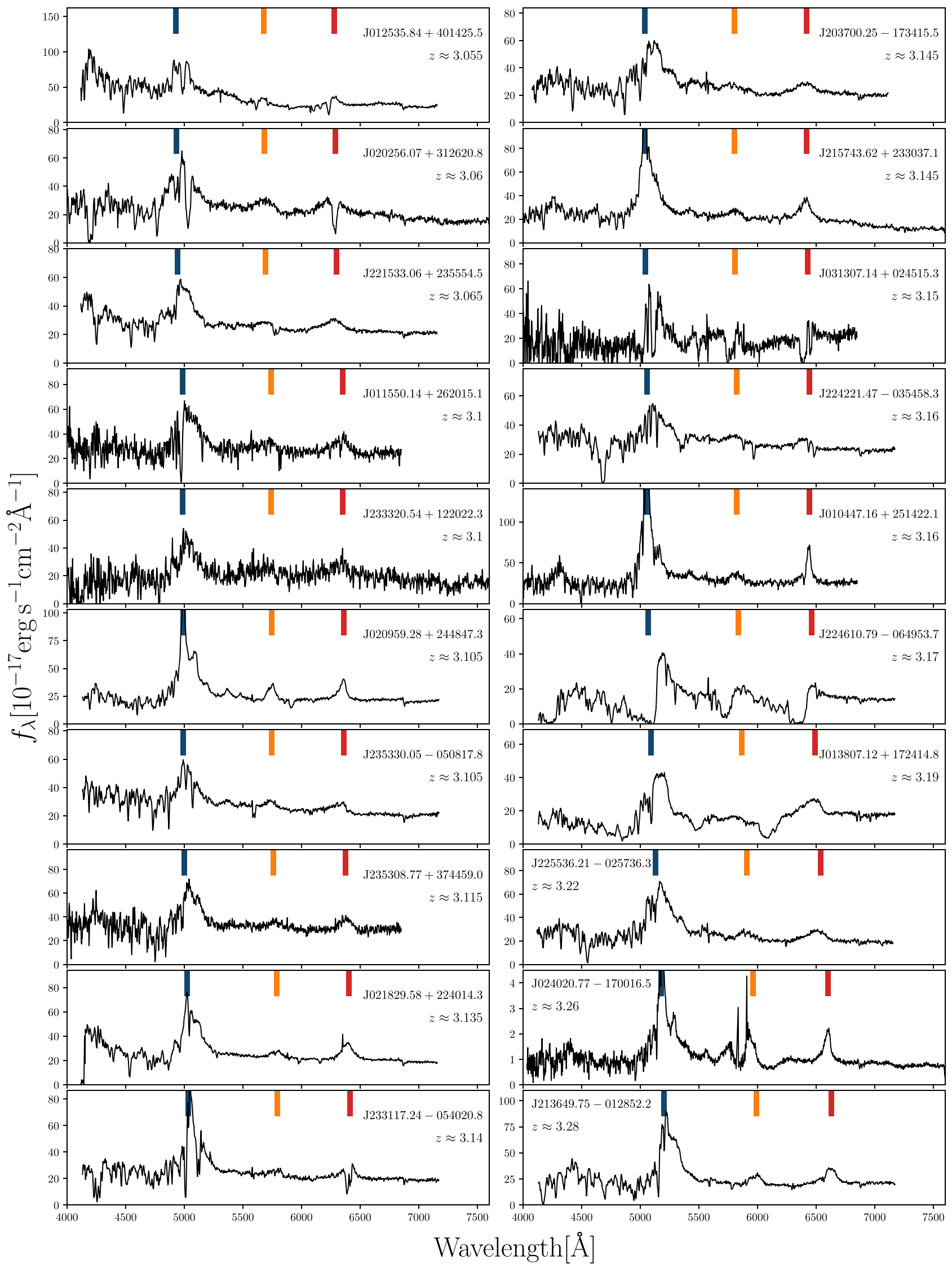

Figure 9. (Continued.) 


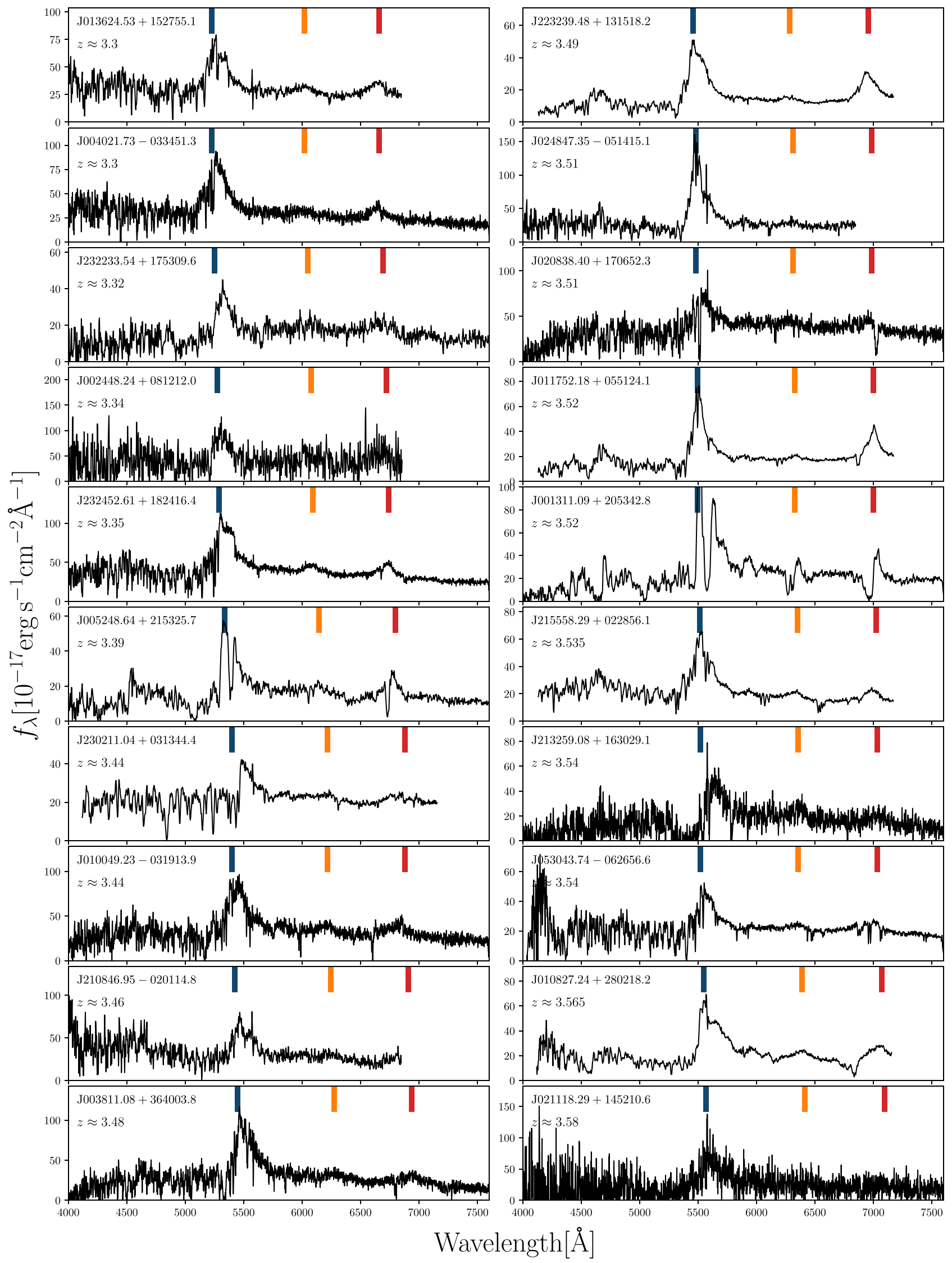

Figure 9. (Continued.) 


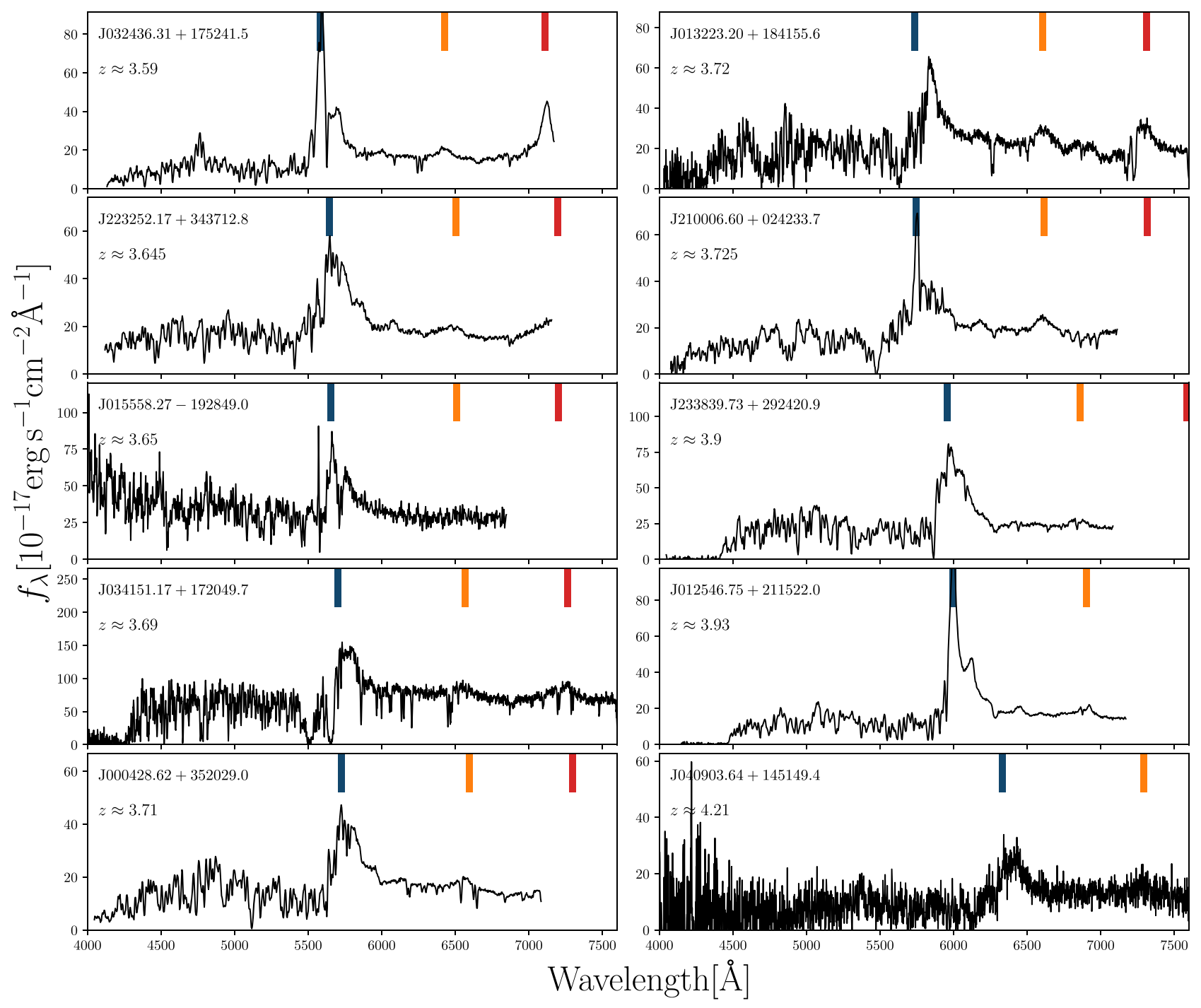

Figure 9. (Continued.)

\section{Appendix C \\ Discovery Spectra of Primary ELQS Quasar Candidates with $z<2.8$}

We present the discovery spectra and general properties for all quasars at $z<2.8$, which were discovered as part of the ELQS, in Figure 10 and Table 10. 


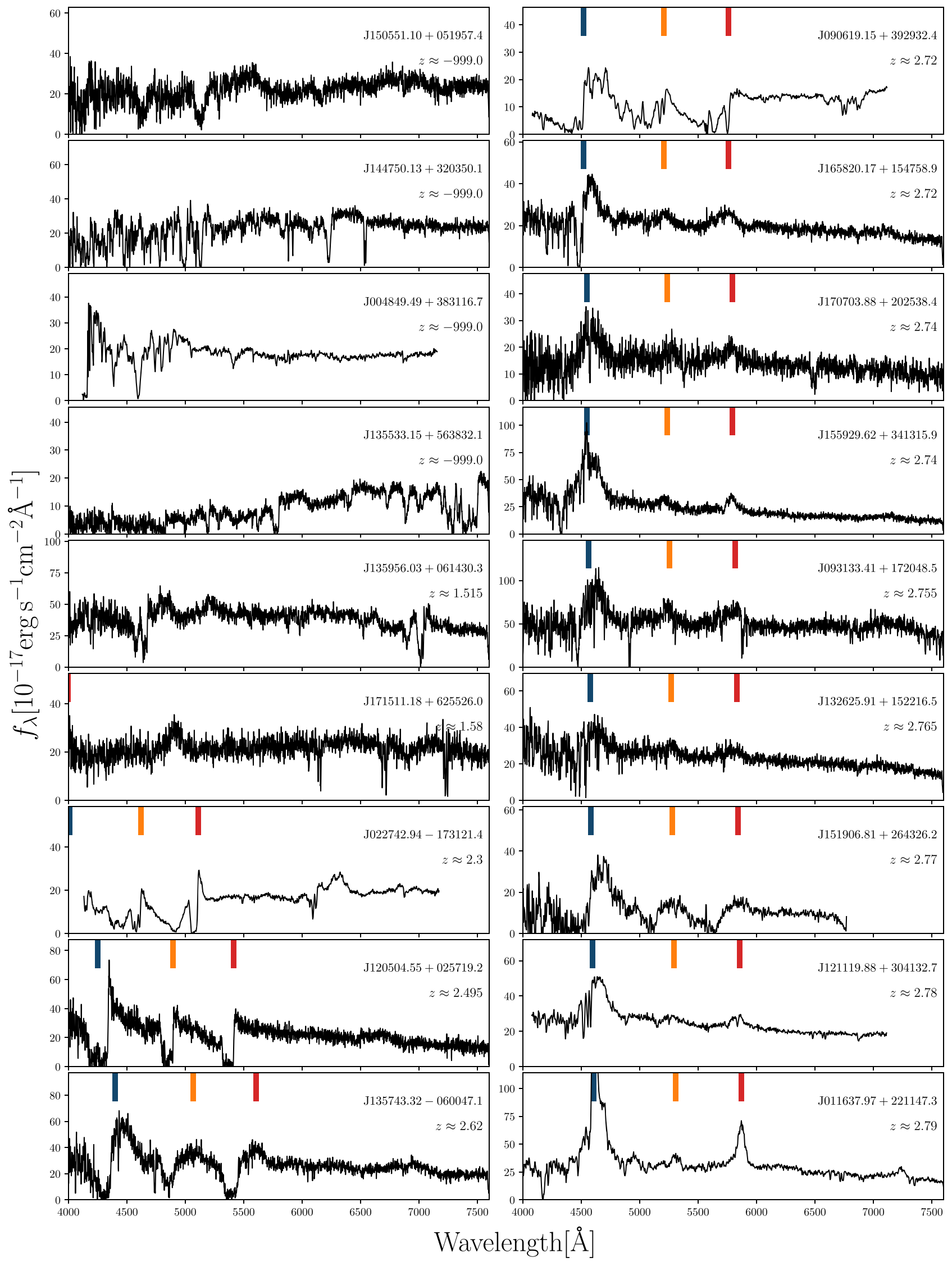

Figure 10. The discovery spectra of primary ELQS candidates at $z<2.8$. The dark blue, orange, and red bars denote the center positions of the broad Ly $\alpha$, Si IV, and C IV emission lines according to the spectroscopic redshift. 
Table 10

Newly Discovered Quasars at $z<2.8$ in the ELQS Sample

\begin{tabular}{|c|c|c|c|c|c|c|c|}
\hline $\begin{array}{l}\text { R.A.(J2000) } \\
\text { (hh:mm:ss.sss) }\end{array}$ & $\begin{array}{l}\text { Decl.(J2000) } \\
\text { (dd:mm:ss.ss) }\end{array}$ & $\begin{array}{c}m_{\mathrm{i}} \\
(\mathrm{mag})\end{array}$ & $\begin{array}{l}M_{1450} \\
\text { (mag) }\end{array}$ & $\begin{array}{c}\text { Spectroscopic } \\
\text { Redshift }\end{array}$ & $\begin{array}{l}\text { Near UV }{ }^{\mathrm{a}} \\
\quad(\mathrm{mag})\end{array}$ & $\begin{array}{l}\text { Far UV } \\
(\mathrm{mag})\end{array}$ & Notes \\
\hline $00: 48: 49.500$ & $+38: 31: 16.73$ & $17.77 \pm 0.03$ & $\cdots$ & $?$ & $\cdots$ & $\cdots$ & 171020 \\
\hline 01:16:37.968 & $+22: 11: 47.31$ & $17.40 \pm 0.02$ & -27.66 & 2.790 & $\cdots$ & $\cdots$ & 170825 \\
\hline $02: 27: 42.939$ & $-17: 31: 21.54$ & $17.58 \pm 0.02$ & -27.12 & 2.300 & $\ldots$ & $\cdots$ & 171021 \\
\hline 09:06:19.161 & $+39: 29: 32.35$ & $17.95 \pm 0.02$ & -27.09 & 2.720 & $21.00 \pm 0.33$ & $\cdots$ & 170517 \\
\hline $09: 31: 33.416$ & $+17: 20: 48.64$ & $16.79 \pm 0.01$ & -28.27 & 2.755 & $\ldots$ & $\cdots$ & 161122 \\
\hline $12: 05: 04.556$ & $+02: 57: 19.20$ & $17.75 \pm 0.01$ & -27.17 & 2.495 & $22.44 \pm 0.19$ & $\cdots$ & 160311 \\
\hline 12:11:19.771 & $+30: 41: 33.25$ & $17.75 \pm 0.02$ & -27.31 & 2.780 & $\ldots$ & $\cdots$ & 170518 \\
\hline $13: 26: 25.921$ & $+15: 22: 16.49$ & $17.62 \pm 0.02$ & -27.43 & 2.765 & $\cdots$ & $\cdots$ & 170405 \\
\hline $13: 55: 33.171$ & $+56: 38: 32.25$ & $17.90 \pm 0.02$ & $\ldots$ & $?$ & $\cdots$ & $\cdots$ & 170503 \\
\hline $13: 57: 43.325$ & $-06: 00: 47.14$ & $17.51 \pm 0.01$ & -27.47 & 2.620 & $\ldots$ & $\ldots$ & 170405 \\
\hline $13: 59: 56.032$ & $+06: 14: 30.19$ & $17.03 \pm 0.02$ & -26.66 & 1.515 & $22.52 \pm 0.18$ & $\cdots$ & 170404 \\
\hline $14: 47: 50.137$ & $+32: 03: 50.29$ & $17.19 \pm 0.01$ & $\ldots$ & $?$ & $\ldots$ & $\cdots$ & 170503 \\
\hline 15:05:51.111 & $+05: 19: 57.32$ & $17.43 \pm 0.01$ & $\ldots$ & $?$ & $\cdots$ & $\cdots$ & 170504 \\
\hline $15: 19: 06.817$ & $+26: 43: 26.29$ & $17.96 \pm 0.01$ & -27.10 & 2.770 & $\cdots$ & $\cdots$ & 150509 \\
\hline $15: 59: 29.631$ & $+34: 13: 16.12$ & $17.84 \pm 0.02$ & -27.21 & 2.740 & $\ldots$ & $\cdots$ & 170406 \\
\hline $16: 58: 20.175$ & $+15: 47: 58.97$ & $17.87 \pm 0.01$ & -27.16 & 2.720 & $\ldots$ & $\cdots$ & 170405 \\
\hline $17: 07: 03.862$ & $+20: 25: 39.27$ & $18.00 \pm 0.04$ & -27.05 & 2.740 & $\ldots$ & $\cdots$ & 170419 \\
\hline $17: 15: 11.188$ & $+62: 55: 26.10$ & $17.58 \pm 0.02$ & -26.14 & 1.580 & $\ldots$ & $\cdots$ & 170503 \\
\hline
\end{tabular}

Note.

a The near- and far-UV magnitudes were obtained from cross-matches within 2 ". 0 to the GALEX GR6/7 data release.

\section{Appendix D \\ Additional Quasars Discovered as Part of This Project}

We present the discovery spectra (Figure 11) and general properties (Table 11) of quasars, which were discovered during exploratory observing runs. 




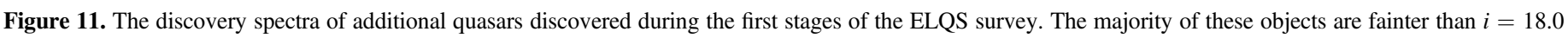

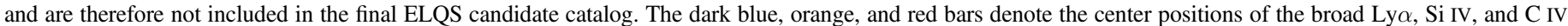
emission lines according to the spectroscopic redshift. 


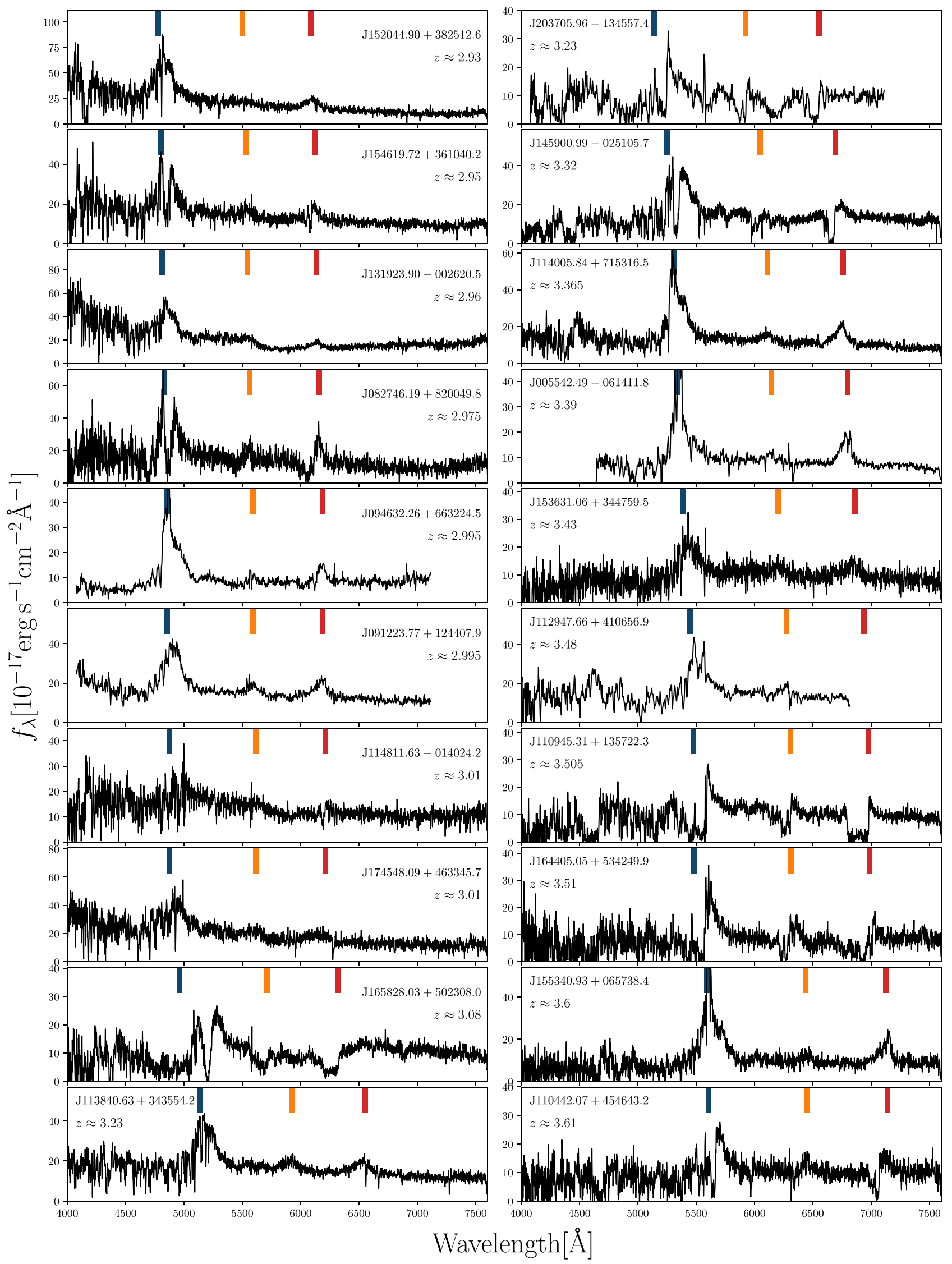

Figure 11. (Continued.) 


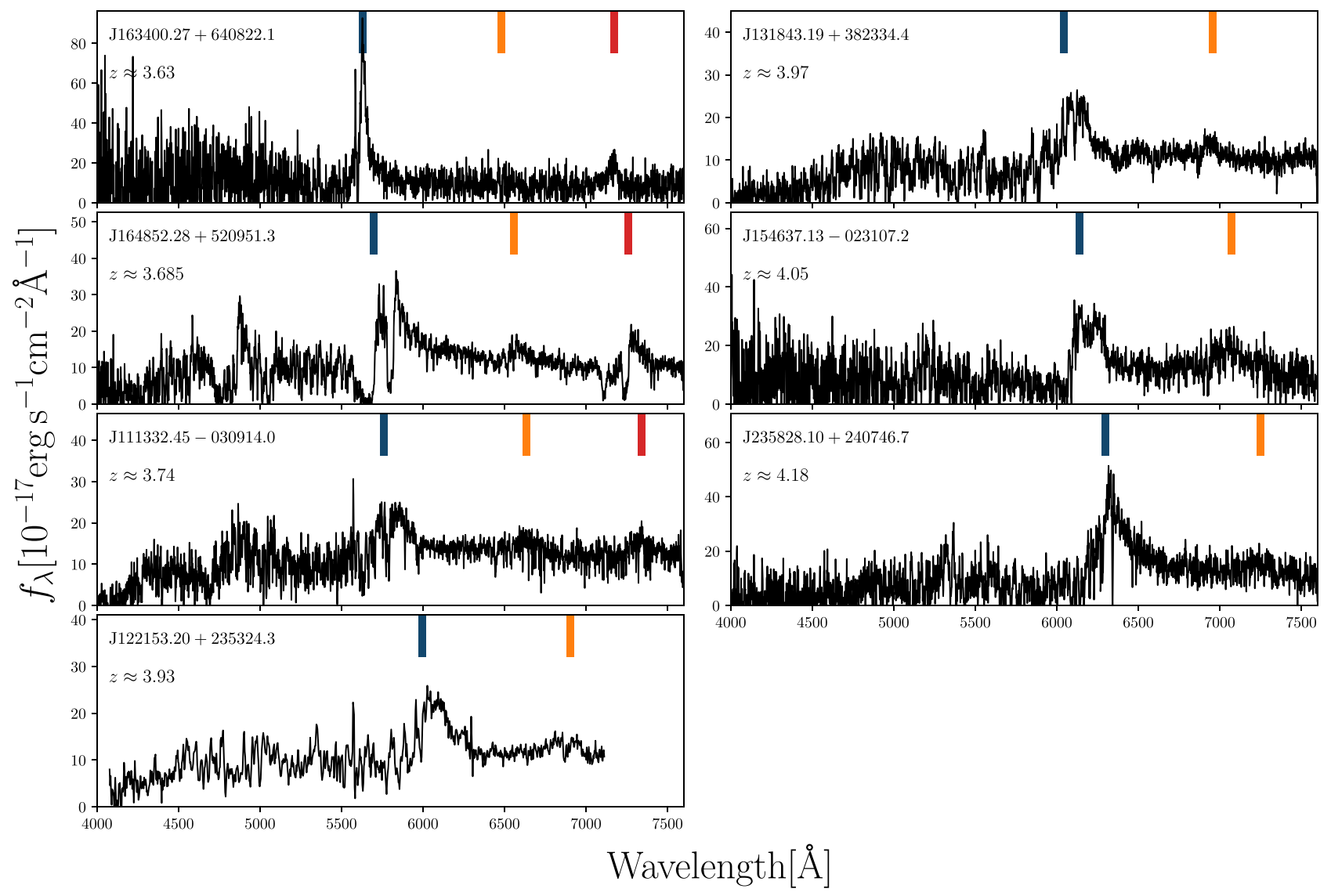

Figure 11. (Continued.)

Table 11

Newly Discovered Quasars Not Part of the ELQS Primary Candidate Catalog

\begin{tabular}{|c|c|c|c|c|c|}
\hline $\begin{array}{l}\text { R.A.(J2000) } \\
\text { (hh:mm:ss.sss) }\end{array}$ & $\begin{array}{l}\text { Decl.(J2000) } \\
\text { (dd:mm:ss.ss) }\end{array}$ & $\begin{array}{c}m_{i} \\
(\mathrm{mag})\end{array}$ & $\begin{array}{l}M_{1450} \\
(\mathrm{mag})\end{array}$ & $\begin{array}{l}\text { Spectroscopic } \\
\text { Redshift }\end{array}$ & Notes \\
\hline $00: 55: 42.504$ & $-06: 14: 11.99$ & $18.43 \pm 0.02$ & -27.06 & 3.390 & 151201 \\
\hline 01:26:30.629 & $+08: 32: 25.35$ & $17.34 \pm 0.01$ & -27.71 & 2.770 & 161115 \\
\hline 02:19:35.796 & $+19: 02: 55.17$ & $17.21 \pm 0.01$ & -27.83 & 2.730 & 161122 \\
\hline 02:41:23.499 & $-07: 05: 12.36$ & $17.38 \pm 0.02$ & -27.67 & 2.770 & 161123 \\
\hline 03:06:56.870 & $-07: 54: 28.60$ & $18.20 \pm 0.02$ & $\ldots$ & $?$ & 151012 \\
\hline 04:41:55.219 & $-01: 03: 49.98$ & $17.22 \pm 0.01$ & $\ldots$ & $?$ & 161123 \\
\hline 05:15:54.937 & $+01: 22: 42.59$ & $18.17 \pm 0.01$ & -26.92 & 2.820 & 161123 \\
\hline 08:06:36.114 & $+48: 40: 26.69$ & $17.30 \pm 0.02$ & -27.70 & 2.680 & 161218 \\
\hline 08:27:46.205 & $+82: 00: 49.95$ & $18.16 \pm 0.02$ & -27.05 & 2.975 & 170405 \\
\hline $09: 12: 23.780$ & $+12: 44: 08.14$ & $18.19 \pm 0.02$ & -27.02 & 2.995 & 170518 \\
\hline 09:46:32.282 & $+66: 32: 24.61$ & $18.36 \pm 0.02$ & -26.85 & 2.995 & 170517 \\
\hline 11:04:42.076 & $+45: 46: 43.26$ & $18.21 \pm 0.02$ & -27.34 & 3.610 & 170419 \\
\hline 11:09:45.306 & $+13: 57: 22.20$ & $18.14 \pm 0.02$ & -27.38 & 3.505 & 170419 \\
\hline $11: 13: 32.451$ & $-03: 09: 14.07$ & $18.05 \pm 0.01$ & -27.60 & 3.740 & 170418 \\
\hline $11: 28: 30.664$ & $+75: 15: 20.94$ & $18.21 \pm 0.02$ & -26.88 & 2.810 & 170406 \\
\hline 11:29:47.663 & $+41: 06: 57.05$ & $18.18 \pm 0.02$ & -27.33 & 3.480 & 150508 \\
\hline 11:38:40.635 & $+34: 35: 54.25$ & $18.01 \pm 0.02$ & -27.36 & 3.230 & 170418 \\
\hline 11:40:05.747 & $+71: 53: 16.17$ & $18.27 \pm 0.03$ & -27.19 & 3.365 & 170405 \\
\hline 11:48:11.638 & $-01: 40: 24.55$ & $18.28 \pm 0.02$ & -26.93 & 3.010 & 170419 \\
\hline $12: 01: 15.165$ & $+30: 13: 58.47$ & $17.97 \pm 0.02$ & -26.90 & 2.460 & 160312 \\
\hline 12:01:16.305 & $+26: 16: 11.89$ & $18.07 \pm 0.02$ & $\ldots$ & $?$ & 170417 \\
\hline $12: 21: 53.197$ & $+23: 53: 24.39$ & $18.09 \pm 0.03$ & -27.67 & 3.930 & 170518 \\
\hline $12: 43: 40.542$ & $+24: 01: 42.14$ & $18.12 \pm 0.02$ & -26.97 & 2.830 & 160312 \\
\hline $13: 14: 17.573$ & $+38: 45: 17.13$ & $18.11 \pm 0.02$ & -26.94 & 2.760 & 170406 \\
\hline $13: 18: 43.193$ & $+38: 23: 34.36$ & $18.11 \pm 0.02$ & -27.66 & 3.970 & 160312 \\
\hline $13: 19: 23.907$ & $-00: 26: 20.55$ & $18.21 \pm 0.01$ & -26.98 & 2.960 & 170417 \\
\hline $13: 58: 58.050$ & $-03: 29: 08.33$ & $18.41 \pm 0.02$ & $\ldots$ & $?$ & 150422 \\
\hline
\end{tabular}


Table 11

(Continued)

\begin{tabular}{|c|c|c|c|c|c|}
\hline $\begin{array}{l}\text { R.A.(J2000) } \\
\text { (hh:mm:ss.sss) }\end{array}$ & $\begin{array}{l}\text { Decl.(J2000) } \\
\text { (dd:mm:ss.ss) }\end{array}$ & $\begin{array}{c}m_{i} \\
(\mathrm{mag})\end{array}$ & $\begin{array}{l}M_{1450} \\
(\mathrm{mag})\end{array}$ & $\begin{array}{c}\text { Spectroscopic } \\
\text { Redshift }\end{array}$ & Notes \\
\hline $14: 23: 57.190$ & $-18: 53: 47.33$ & $18.36 \pm 0.02$ & -25.81 & 1.930 & 170518 \\
\hline 14:59:01.000 & $-02: 51: 05.81$ & $18.01 \pm 0.01$ & -27.43 & 3.320 & 160310 \\
\hline $15: 20: 44.943$ & $+38: 25: 12.27$ & $18.07 \pm 0.01$ & -27.11 & 2.930 & 170406 \\
\hline $15: 36: 31.102$ & $+34: 47: 59.58$ & $18.35 \pm 0.02$ & -27.14 & 3.430 & 160312 \\
\hline $15: 46: 19.728$ & $+36: 10: 40.41$ & $18.21 \pm 0.02$ & -26.98 & 2.950 & 170406 \\
\hline $15: 46: 37.131$ & $-02: 31: 07.62$ & $18.14 \pm 0.02$ & -27.68 & 4.050 & $170418^{\mathrm{a}}$ \\
\hline 15:53:40.946 & $+06: 57: 38.44$ & $18.05 \pm 0.02$ & -27.51 & 3.600 & 170404 \\
\hline 16:04:52.284 & $+38: 47: 55.06$ & $17.40 \pm 0.02$ & -27.66 & 2.800 & 160311 \\
\hline $16: 21: 33.452$ & $+43: 46: 28.41$ & $17.72 \pm 0.01$ & -27.32 & 2.740 & $160313^{\mathrm{a}}$ \\
\hline $16: 34: 00.284$ & $+64: 08: 22.10$ & $18.31 \pm 0.02$ & -27.25 & 3.630 & 170406 \\
\hline $16: 35: 25.313$ & $+38: 14: 29.42$ & $18.25 \pm 0.01$ & -26.83 & 2.790 & 170419 \\
\hline $16: 40: 03.561$ & $+53: 26: 33.67$ & $18.31 \pm 0.01$ & -27.02 & 3.165 & 170419 \\
\hline 16:44:05.048 & $+53: 42: 49.90$ & $18.39 \pm 0.02$ & -27.12 & 3.510 & 170419 \\
\hline $16: 48: 52.294$ & $+52: 09: 51.37$ & $18.13 \pm 0.01$ & -27.47 & 3.685 & $170406^{\mathrm{a}}$ \\
\hline $16: 58: 28.042$ & $+50: 23: 07.98$ & $18.09 \pm 0.01$ & -27.17 & 3.080 & 170406 \\
\hline 17:08:44.725 & $+28: 27: 30.49$ & $17.60 \pm 0.01$ & -27.54 & 2.890 & 160312 \\
\hline $17: 17: 21.347$ & $+42: 24: 28.12$ & $18.17 \pm 0.02$ & -27.35 & 3.490 & 170419 \\
\hline $17: 45: 48.100$ & $+46: 33: 45.71$ & $18.03 \pm 0.03$ & -27.19 & 3.010 & 160313 \\
\hline 20:37:05.975 & $-13: 45: 57.50$ & $18.07 \pm 0.03$ & -27.30 & 3.230 & 170518 \\
\hline 20:59:07.574 & $+08: 36: 44.19$ & $18.26 \pm 0.02$ & -27.07 & 3.170 & 170518 \\
\hline $22: 11: 24.148$ & $+25: 43: 27.16$ & $16.94 \pm 0.03$ & -28.14 & 2.805 & 161115 \\
\hline 22:51:59.484 & $+17: 28: 44.64$ & $17.22 \pm 0.01$ & $\ldots$ & $?$ & 171110 \\
\hline $22: 56: 43.450$ & $-02: 46: 32.83$ & $17.65 \pm 0.01$ & -27.16 & 2.400 & 151011 \\
\hline 23:58:28.105 & $+24: 07: 46.70$ & $18.01 \pm 0.01$ & -27.90 & 4.180 & 161123 \\
\hline
\end{tabular}

Note.

${ }^{\mathrm{a}}$ These objects were also independently discovered by Yang et al.

\section{ORCID iDs}

Jan-Torge Schindler (i) https://orcid.org/0000-00024544-8242

Xiaohui Fan (1) https://orcid.org/0000-0003-3310-0131

Ian D. McGreer (1) https://orcid.org/0000-0002-3461-5228

Jinyi Yang (i) https://orcid.org/0000-0001-5287-4242

Feige Wang (il https://orcid.org/0000-0002-7633-431X

Johan P. U. Fynbo (1) https://orcid.org/0000-0002-8149-8298

Jens-Kristian Krogager (1) https://orcid.org/0000-00024912-9388

Elisabeth M. Green (ㄷ) https://orcid.org/0000-0003-3688-2298

Yun-Hsin Huang (1) https://orcid.org/0000-0003-4955-5632

Jennifer Kadowaki i https://orcid.org/0000-0002-3767-9681

Ya-Lin Wu (1) https://orcid.org/0000-0002-4392-1446

Minghao Yue (1) https://orcid.org/0000-0002-5367-8021

\section{References}

Abazajian, K. N., Adelman-McCarthy, J. K., Agüeros, M. A., et al. 2009, ApJS, 182, 543

Akiyama, M., He, W., Ikeda, H., et al. 2018, PASJ, 70, S34

Allen, J. T., Hewett, P. C., Maddox, N., Richards, G. T., \& Belokurov, V. 2011, MNRAS, 410, 860

Astropy Collaboration, Robitaille, T. P., Tollerud, E. J., et al. 2013, A\&A, 558, A33

Avni, Y., \& Bahcall, J. N. 1980, ApJ, 235, 694

Bahcall, J. N., Maoz, D., Schneider, D. P., Yanny, B., \& Doxsey, R. 1992, ApJL, 392, L1

Bañados, E., Venemans, B. P., Mazzucchelli, C., et al. 2018, Natur, 553, 473 Becker, R. H., White, R. L., \& Helfand, D. J. 1995, ApJ, 450, 559

Blanton, M. R., Bershady, M. A., Abolfathi, B., et al. 2017, AJ, 154, 28

Boller, T., Freyberg, M. J., Trümper, J., et al. 2016, A\&A, 588, A103

Boyle, B. J., Shanks, T., Croom, S. M., et al. 2000, MNRAS, 317, 1014
Boyle, B. J., Shanks, T., \& Peterson, B. A. 1988, MNRAS, 235, 935

Breiman, L. 2001, Machine Learning, 45, 5

Croom, S. M., Richards, G. T., Shanks, T., et al. 2009, MNRAS, 399, 1755

Dai, X., Shankar, F., \& Sivakoff, G. R. 2008, ApJ, 672, 108

Dawson, K. S., Kneib, J.-P., Percival, W. J., et al. 2016, AJ, 151, 44

Dawson, K. S., Schlegel, D. J., Ahn, C. P., et al. 2013, AJ, 145, 10

Dong, X. Y., Wu, X.-B., Ai, Y. L., et al. 2018, AJ, 155, 189

Efron, B., \& Petrosian, V. 1992, ApJ, 399, 345

Eisenstein, D. J., Weinberg, D. H., Agol, E., et al. 2011, AJ, 142, 72

Fan, X., Strauss, M. A., Schneider, D. P., et al. 2001, AJ, 121, 54

Flesch, E. W. 2015, PASA, 32, e010

Foreman-Mackey, D., Hogg, D. W., Lang, D., \& Goodman, J. 2013, PASP, 125,306

Glikman, E., Djorgovski, S. G., Stern, D., et al. 2011, ApJL, 728, L26

Górski, K. M., Hivon, E., Banday, A. J., et al. 2005, ApJ, 622, 759

Hall, P. B., Anderson, S. F., Strauss, M. A., et al. 2002, ApJS, 141, 267

Hunter, J. D. 2007, CSE, 9, 90

Ibata, R. A., Lewis, G. F., Irwin, M. J., Lehár, J., \& Totten, E. J. 1999, AJ, 118, 1922

Inada, N., Oguri, M., Shin, M.-S., et al. 2012, AJ, 143, 119

Ivezić, Ž., Connelly, A. J., Vander Plas, J. T., \& Gray, A. 2014, Statistics, Data Mining, and Machine Learning in Astronomy (Princeton, NJ: Princeton Univ. Press)

Jiang, L., Fan, X., Annis, J., et al. 2008, AJ, 135, 1057

Jiang, L., Fan, X., Ivezić, Ž., et al. 2007, ApJ, 656, 680

Jiang, L., McGreer, I. D., Fan, X., et al. 2016, ApJ, 833, 222

Jones, E., Oliphant, T., Peterson, P., et al. 2001, SciPy: Open source scientific tools for Python, https://www.scipy.org/

Koo, D. C., \& Kron, R. G. 1988, ApJ, 325, 92

Kormendy, J., \& Ho, L. C. 2013, ARA\&A, 51, 511

Kulkarni, G., Worseck, G., \& Hennawi, J. F. 2018, MNRAS, submitted (arXiv: 1807.09774)

Lampton, M., Margon, B., \& Bowyer, S. 1976, ApJ, 208, 177

Lynden-Bell, D. 1971, MNRAS, 155, 95

Maddox, N., Hewett, P. C., Warren, S. J., \& Croom, S. M. 2008, MNRAS, 386,1605

Magain, P., Surdej, J., Vanderriest, C., Pirenne, B., \& Hutsemekers, D. 1992, A\&A, 253, L13 
Maloney, A., \& Petrosian, V. 1999, ApJ, 518, 32

Marshall, H. L., Tananbaum, H., Avni, Y., \& Zamorani, G. 1983, ApJ, 269, 35

Martin, D. C., Fanson, J., Schiminovich, D., et al. 2005, ApJL, 619, L1

Masters, D., Capak, P., Salvato, M., et al. 2012, ApJ, 755, 169

Matsuoka, Y., Strauss, M. A., Kashikawa, N., et al. 2018, ApJ, 869, 150

McGreer, I. D., Fan, X., Jiang, L., \& Cai, Z. 2018, AJ, 155, 131

McGreer, I. D., Jiang, L., Fan, X., et al. 2013, ApJ, 768, 105

McKinney, W. 2010, in Proc. of the 9th Python in Science Conf., 51

Mortlock, D. J., Warren, S. J., Venemans, B. P., et al. 2011, Natur, 474, 616

Newville, M., Stensitzki, T., Allen, D. B., \& Ingargiola, A. 2014, LMFIT: NonLinear Least-Square Minimization and Curve-Fitting for Python, Zenodo, doi:10.5281/zenodo. 11813

Oke, J. B., \& Gunn, J. E. 1983, ApJ, 266, 713

Oliphant, T. E. 2007, CSE, 9, 10

Onoue, M., Kashikawa, N., Willott, C. J., et al. 2017, ApJL, 847, L15

Page, M. J., \& Carrera, F. J. 2000, MNRAS, 311, 433

Palanque-Delabrouille, N., Magneville, C., Yèche, C., et al. 2016, A\&A, 587, A41

Pâris, I., Petitjean, P., Aubourg, É, et al. 2018, A\&A, 613, A51

Pâris, I., Petitjean, P., Ross, N. P., et al. 2017, A\&A, 597, A79

Patnaik, A. R., Browne, I. W. A., Walsh, D., Chaffee, F. H., \& Foltz, C. B. 1992, MNRAS, 259, $1 \mathrm{P}$

Pedregosa, F., Varoquaux, G., Gramfort, A., et al. 2011, Journal of Machine Learning Research, 12, 2825

Pei, Y. C. 1995, ApJ, 438, 623

Planck Collaboration, Ade, P. A. R., Aghanim, N., et al. 2016, A\&A, 594, A13

Prochaska, J. X., Herbert-Fort, S., \& Wolfe, A. M. 2005, ApJ, 635, 123

Richards, G. T., Strauss, M. A., Fan, X., et al. 2006, AJ, 131, 2766

Ross, N. P., McGreer, I. D., White, M., et al. 2013, ApJ, 773, 14

Salvato, M., Buchner, J., Budavári, T., et al. 2018, MNRAS, 473, 4937

Schindler, J.-T., Fan, X., McGreer, I. D., et al. 2017, ApJ, 851, 13
Schindler, J.-T., Fan, X., McGreer, I. D., et al. 2018, ApJ, 863, 144

Schlafly, E. F., \& Finkbeiner, D. P. 2011, ApJ, 737, 103

Schmidt, M. 1968, ApJ, 151, 393

Schmidt, M., Schneider, D. P., \& Gunn, J. E. 1995, AJ, 110, 68

Schmidt, T. M., Worseck, G., Hennawi, J. F., Prochaska, J. X., \& Crighton, N. H. M. 2017, ApJ, 847, 81

Schneider, D. P., Richards, G. T., Hall, P. B., et al. 2010, AJ, 139, 2360

Simcoe, R. A., Sargent, W. L. W., \& Rauch, M. 2004, ApJ, 606, 92

Skrutskie, M. F., Cutri, R. M., Stiening, R., et al. 2006, AJ, 131, 1163

Tody, D. 1986, Proc. SPIE, 627, 733

Tody, D. 1993, in ASP Conf. Ser. 52, Astronomical Data Analysis Software and Systems II, ed. R. J. Hanisch, R. J. V. Brissenden, \& J. Barnes (San Francisco, CA: ASP), 173

Truemper, J. 1982, AdSpR, 2, 241

Trump, J. R., Hall, P. B., Reichard, T. A., et al. 2006, ApJS, 165, 1

Vanden Berk, D. E., Richards, G. T., Bauer, A., et al. 2001, AJ, 122, 549

Wang, F., Yang, J., Fan, X., et al. 2018, ApJ, submitted (arXiv:1810.11926)

Weymann, R. J., Morris, S. L., Foltz, C. B., \& Hewett, P. C. 1991, ApJ, 373,23

Willott, C. J., Delorme, P., Reylé, C., et al. 2010, AJ, 139, 906

Worseck, G., \& Prochaska, J. X. 2011, ApJ, 728, 23

Worseck, G., Prochaska, J. X., Hennawi, J. F., \& McQuinn, M. 2016, ApJ, 825,144

Wright, E. L., Eisenhardt, P. R. M., Mainzer, A. K., et al. 2010, AJ, 140, 1868

Wu, X.-B., Hao, G., Jia, Z., Zhang, Y., \& Peng, N. 2012, AJ, 144, 49

Wu, X.-B., \& Jia, Z. 2010, MNRAS, 406, 1583

Yang, J., Wang, F., Wu, X.-B., et al. 2016, ApJ, 829, 33

York, D. G., Adelman, J., Anderson, J. E., Jr., et al. 2000, AJ, 120, 1579

Zhao, G., Zhao, Y.-H., Chu, Y.-Q., Jing, Y.-P., \& Deng, L.-C. 2012, RAA, 12,723

Zheng, W., Syphers, D., Meiksin, A., et al. 2015, ApJ, 806, 142 